\title{
The vacuum ultraviolet spectrum of cyclohepta-1, 3, 5-triene: analysis of the singlet and triplet excited states by $a b$ initio and density functional methods.
}

\author{
Michael H. Palmer, ${ }^{1, a}$ Søren Vrønning Hoffmann, ${ }^{2, a}$ Nykola C. Jones, ${ }^{2, a}$ \\ Marcello Coreno, ${ }^{3, a}$ Monica de Simone ${ }^{4, a}$, Cesare Grazioli, ${ }^{4, a}$ and R. Alan \\ Aitken, ${ }^{5, \mathrm{a}}$ \\ ${ }^{1}$ School of Chemistry, University of Edinburgh, Joseph Black Building, David Brewster \\ Road, Edinburgh EH9 3FJ, Scotland, UK \\ ${ }^{2}$ ISA, Department of Physics and Astronomy, Aarhus University, Ny Munkegade 120, \\ DK-8000 Aarhus C, Denmark \\ ${ }^{3}$ ISM-CNR, Istituto di Struttura della Materia, LD2 Unit 34149 Trieste, Italy, \\ ${ }^{4}$ IOM-CNR , Istituto Officina dei Materiali, Elettra-Sincrotrone, I-34149 Basovizza, Trieste S.S., \\ Trieste, Italy \\ ${ }^{5}$ School of Chemistry, University of St Andrews, North Haugh, St Andrews Fife, KY16 9ST, \\ Scotland, UK \\ a) Electronic addresses: m.h.palmer@ed.ac.uk; vronning@phys.au.dk; nykj@phys.au.dk; \\ marcello.coreno@elettra.eu; desimone@iom.cnr.it; grazioli@ism.cnr.it; raa@st-andrews.ac.uk
}

\begin{abstract}
The vacuum ultraviolet spectrum (VUV) for cyclohepta-1,3,5-triene (CHT) up to $10.8 \mathrm{eV}$ shows several broad bands, which are compared with electron impact spectra. Local curve fitting exposed groups of sharp vibrational peaks, which are assigned to Rydberg states. The vertical excitation profile of the VUV spectrum, reproduced by time dependent density functional theory (TDDFT), gives a good interpretation of the principal regions of absorption. Fourth order Möller-Plessett perturbation theory, including single, double and quadruple excitations (MP4(SDQ)), showed that the lowest singlet and triplet states retain $\mathrm{C}_{\mathrm{s}}$ symmetry. This contrasts with TDDFT where several low-lying excited states are planar. Detailed vibrational analysis of the first UV band was performed by Franck-Condon (FC), HerzbergTeller (HT), and their combined methods (FCHT). These show the dominance of mid-range
\end{abstract}


frequencies, while the lowest frequency $\left(75 \mathrm{~cm}^{-1}\right)$ has negligible importance. In contrast, the second excited (Rydberg) state shows a major progression with separations $115(6) \mathrm{cm}^{-1}$. This is interpreted by re-analysis of the $\mathrm{X}^{2} \mathrm{~A}^{\prime}$ ionic state at the anharmonic level. Extremely low exponent Gaussian functions enabled several low-lying Rydberg state energies to be determined theoretically; extrapolation of the $3 \mathrm{~s}, 4 \mathrm{~s}$ and $5 \mathrm{~s}$ Rydberg state calculated energies gives the adiabatic ionization energy (AIE) as $7.837 \mathrm{eV}(4)$ with $\delta 0.964$ (2). Similarly, extrapolation of the centroids of the observed Rydberg states gave the vertical ionization energy (VIE) as $\mathrm{VIE}_{1}=8.675 \pm 0.077$, close to the photoelectron spectral (PES) vertical ionization energy value $(8.55(1) \mathrm{eV})$.

\section{INTRODUCTION}

Recently, we reported a new high-resolution photoelectron (PES) spectrum of cyclohepta-1, 3, 5-triene $\left(\mathrm{C}_{7} \mathrm{H}_{8}\right.$, here abbreviated to CHT). ${ }^{1}$ We now present a synchrotron based, highresolution VUV spectrum of CHT, which is subjected to detailed spectral and theoretical analysis. The CHT molecule undergoes 'boat to boat' interconversions, Cs $\leftrightarrows$ Cs symmetry, as shown in Figure $1(1 \mathrm{~A}+1 \mathrm{~B})$. The overall CHT ring is characterized by 'bow' $(\theta)$ and 'stern' $(\phi)$ angles for the boat, shown in Figure 2. These angles vary considerably with the electronic state. In the $\mathrm{X}^{1} \mathrm{~A}^{\prime}$ ground state, relative to the central $\mathrm{C}_{1} \mathrm{C}_{2} \mathrm{C}_{5} \mathrm{C}_{6}$ plane, the $7-\mathrm{CH}_{2}$ group bow angle $(\theta)$ is $35.6^{\circ}$, while the stern angle $(\phi)$ is $52.9^{\circ}$ for the $\mathrm{C}_{2} \mathrm{C}_{3}=\mathrm{C}_{4} \mathrm{C}_{5}$ group.

Our previous PES study of CHT, ${ }^{1}$ showed an almost complete lack of vibrational structure, even though one very low vibrational frequency was calculated for the $\mathrm{X}^{2} \mathrm{~A}^{\prime}$ state; this will be discussed later. A similar lack of vibrational detail was previously found in the PES of cyclooctatetraene (COT). This was attributed to a related conformational $\mathrm{D}_{2 \mathrm{~d}} \leftrightarrows \mathrm{D}_{2 \mathrm{~d}}$ interconversion (Figure 1, 3A,3B), ${ }^{2,3}$ accompanied by a high density of vibrational states.

Figure 1. Structural relationships between cyclohepta-1,3,5-triene (1A, 1B), norcaradiene $(2 \mathrm{~A}, 2 \mathrm{~B})$ and cyclooctatetraene $(3 \mathrm{~A}, 3 \mathrm{~B})$ 


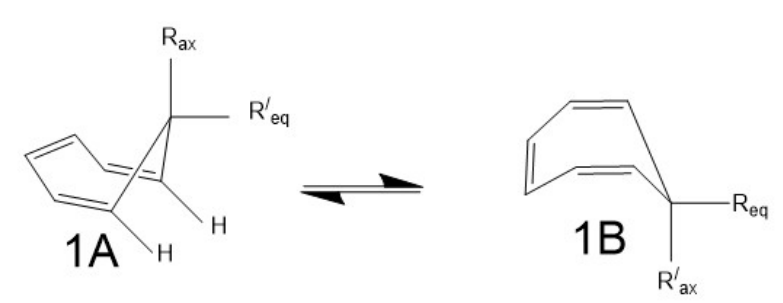

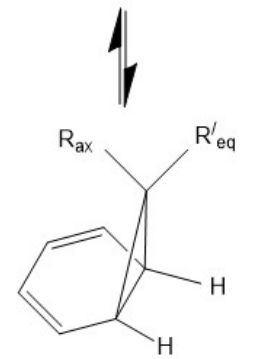

2A

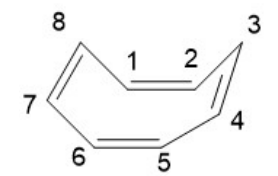

$3 \mathrm{~A}$

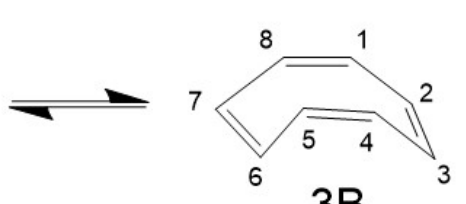

3B

An unexpected discovery during our theoretical study of the CHT ionic states, ${ }^{1}$ was that attempts to generate low-lying CHT states of ${ }^{2} \mathrm{~A}^{\prime \prime}$ symmetry always led, via structural transformation, to ionic states of norcaradiene (NCD); this is shown for the neutral molecules in Figure $1(2 \mathrm{~A}, \mathrm{~B})$. The cross-ring proximity of the classical $\pi$-bonds together with an increased 'bow' angle in the developing ${ }^{2} \mathrm{~A}^{\prime \prime}$ states reduces the $\mathrm{C}_{1}$ to $\mathrm{C}_{6}$ (non-bonded) distance; conrotatory motion in the singly occupied orbital (SOMO) then allows the NCD ring to close to the ${ }^{2} \mathrm{~A}^{\prime /}$ states. Further studies of the energy surfaces for the two lowest CHT ionic states in Cs symmetry, as a function of the non-bonded $\mathrm{C}_{1} \ldots \mathrm{C}_{6}$ distance, showed that ${ }^{2} \mathrm{~A}^{\prime \prime}$ states do exist for a limited range of distance, and exhibit a wide minimum. ${ }^{1}$ This showed that the multiconfiguration self-consistent field (MCSCF) and configuration interaction (CI) methods, which were initiated close to the $\mathrm{X}^{1} \mathrm{~A}^{\prime}$ structure, were unable to locate minima for the $\mathrm{CHT}{ }^{2} \mathrm{~A}^{\prime \prime}$ states close to the equilibrium geometry. The CHT and NCD energy surfaces must be very close in 
certain regions of the hypersurface. Overall, the observed PES for CHT was more consistent with the theoretical PES of CHT than that for NCD. ${ }^{1}$

We now present the first VUV spectrum of CHT, compare with electron impact (EI) profiles, and analyze both spectral types in considerable detail. However, it is critical to ensure that the spectrum we obtain relates to the input CHT sample, since CHT undergoes several thermal and photochemical transformations. We outline these potential hazards to interpretation here.

Several investigations, ${ }^{1,4-9}$ especially by NMR, failed to show the presence of NCD in samples of CHT near room temperature. This apparently excludes the equilibria $1 \leftrightarrows 2$, for both A and $\mathrm{B}$ in Figure 1, and imply that $\mathrm{NCD}$ is a short-lived intermediate ${ }^{10}$ rather than a stable compound. However, CHT and NCD behave as valence tautomers in some thermal and photochemical reactions. For example, interaction of $\mathrm{CHT}$ with maleic anhydride, leads to both endo- and exo-adducts. ${ }^{11,12}$ Reaction rate studies show these reactions are unimolecular in dienophile concentration, and the conclusion must be that the CHT $\leftrightarrows \mathrm{NCD}$ equilibrium is fast relative to adduct formation. ${ }^{11,12}$

CHT undergoes thermal 1,5 and $1,7 \mathrm{H}$-atom migrations leading to an identical molecular species. ${ }^{13}$ It also undergoes rearrangement to toluene (TOL) at elevated temperatures (375 to $\left.450{ }^{\circ} \mathrm{C}\right),{ }^{14}$ and in shock waves at even higher temperatures. ${ }^{15}$ Detailed study of these interconversions have been made using density functional theory (DFT) and complete active space self-consistent field methods (CASSCF). ${ }^{13}$ The CHT to TOL mechanism is $1^{\text {st }}$ order and involvement of NCD seems probable. ${ }^{13}$

Photochemically initiated changes parallel to these thermal ones also occur. UV absorption spectra in the range 185 to $302 \mathrm{~nm}(4.105$ to $6.702 \mathrm{eV})$ for CHT and some alkyl derivatives have been reported by various groups, ${ }^{15-18}$ which demonstrate the potential complexities of CHT photochemistry. 
The lowest UV band of CHT has been of considerable interest in a series of pump-probe (P-P) investigations. ${ }^{16-19}$ Irradiation near the centre of the first band ( $\left.\mathrm{S}_{1}\right)$ of CHT, close to $266 \mathrm{~nm}$ (4.661 eV), leads to nearly complete formation of a vibrationally highly excited ground state $\left(\mathrm{S}_{0} *\right)$ by internal conversion (IC). This has then been exploited by $[1+1]$ multiphoton ionization (MPI) ${ }^{16,17}$ coherent anti-Stokes resonant scattering $(\mathrm{CARS})^{18}$ and a resonance Raman study. ${ }^{19}$ In the MPI study, this makes the ionization probability smaller than in TOL by a factor of $10^{5}$. The difference between the irradiation position and the band onset leads to estimates of the excess vibrational energy between zero and $4300 \mathrm{~cm}^{-1}$. The absence of both fluorescence and vibrational structure on the $\mathrm{S}_{1}$ band, even in a $12^{\circ} \mathrm{K}$ matrix for $\mathrm{CHT}$, shows the $S_{1}$ upper state lifetime is very short, and estimated to be $114 \mathrm{fs} .{ }^{17,18}$

Similarly, following excitation into $\mathrm{S}_{1}$, CHT undergoes a photochemically allowed H-atom $[1,7]$-shift reaction, where one $\mathrm{H}$-atom of the $7-\mathrm{CH}_{2}$ group is transferred to an adjacent carbon atom. ${ }^{19}$ Using the same P-P technique with resonance Raman, at $284 \mathrm{~nm}(4.366 \mathrm{eV})$, gives an appearance time for the isomeric species as 25 ps. ${ }^{19}$ A theoretical study suggests that the initially populated $1^{1} \mathrm{~A}^{\prime \prime}$ state undergoes IC to the close lying ${ }^{1} \mathrm{~A}^{\prime}$ excited state and transfer is completed via two conical intersections. ${ }^{20}$

The structurally different photochemical conversion of CHT to bicyclo[3,2,0]heptadiene $(\mathrm{BCH})$ and small amounts of toluene, probably follows similar steps. Filtered Hg emission at $265.2 \mathrm{~nm}$, was generated from a 100 watt medium pressure mercury arc lamp. ${ }^{21}$ Consistent with the studies above, it was postulated that different high vibrational levels of the CHT ground state were directly involved in the formation of each compound. Irradiation at $313 \mathrm{~nm}$ $(3.961 \mathrm{eV})$ produced less TOL. ${ }^{22}$

We now present the first VUV spectrum of CHT and analyze its profile in considerable detail. This includes comparison of our calculated triplet state energies with two major studies using EI. $^{23,24}$ Electron affinities (EA) for CHT, determined by electron transmission spectroscopy 
(ETS), ${ }^{25,26}$ are included since electron capture is evident in some EI studies. We explore the electronic state sequence using several theoretical methods.

Figure 2. The local planes which include the boat bow and stern angles $(\phi$ and $\theta)$ for the $\mathrm{C}_{2} \mathrm{C}_{3}=\mathrm{C}_{4} \mathrm{C}_{5}$ and $7-\mathrm{CH}_{2}$ groups relative to the central $\mathrm{C}_{1} \mathrm{C}_{2} \mathrm{C}_{5} \mathrm{C}_{6}$ plane. $\mathrm{H}$-atoms are not restricted to local $\mathrm{CC}=\mathrm{CC}$ planes. Reproduced from reference 1 , with the permission of AIP Publishing.

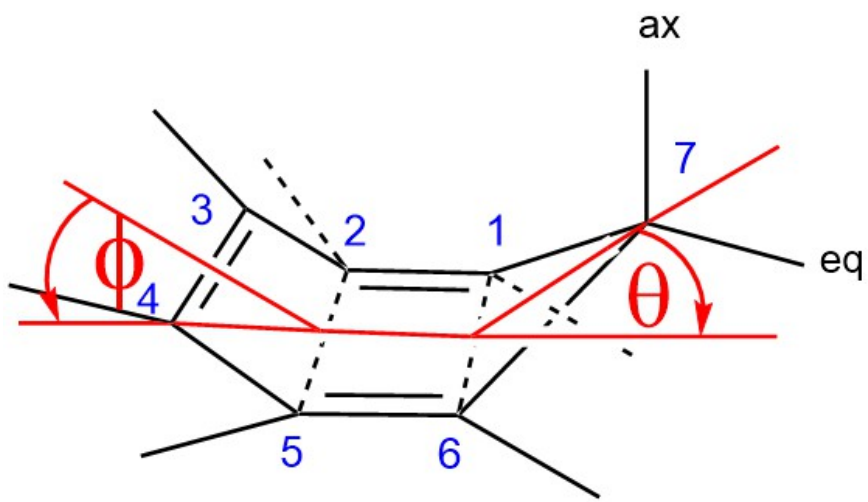

\section{METHODS}

We reported details of the ${ }^{1} \mathrm{H}$ and ${ }^{13} \mathrm{C}$ NMR spectra of the CHT sample previously. ${ }^{1}$ Despite the thermal and photochemical interconversion of CHT above, there is no evidence of anything other than CHT in the VUV spectrum, under the conditions for these measurements; this is discussed further below.

As in our recent COT and CHT studies, ${ }^{1-3}$ we use a combination of theoretical methods, since each of these has specific uses, and no single computational suite can offer us a complete analysis. The ground state of CHT in Cs symmetry has $15 \mathrm{a}^{\prime}$ and $10 \mathrm{a}^{\prime /}$ occupied MOs. In this paper, the core MOs $\left(4 a^{\prime}+3 a^{\prime \prime}\right)$ are omitted, and valence shell numbering with occupied orbital sequence numbers $1-11 a^{\prime}$ and $1-7 a^{/ /}$is used throughout.

1.Theoretical methods. The GAUSSIAN suite (G-16 version) ${ }^{27}$ was used for equilibrium structure determination and vibrational frequencies. Some calculations used density functional theoretical (DFT) methods, including the Becke three-parameter hybrid functionals (B3LYP), ${ }^{28,29}$ including a long-range-corrected exchange functional (CAM-B3LYP). ${ }^{30}$ It 
became apparent that some low-lying triplets and singlets, generated by time dependent density functional theory (TDDFT), adopted a planar $\mathrm{C}_{2 \mathrm{~V}}$ configuration on excitation. This was not borne out by more sophisticated calculations (below); although both $\mathrm{Cs}_{\mathrm{s}}$ and $\mathrm{C}_{2 \mathrm{~V}}$ symmetries were considered for the singlet and triplet states, only the latter are considered in detail.

Highly correlated wave-functions for adiabatic excited states were obtained by using either G$16^{27}$ or the MOLPRO suite ${ }^{31-33}$ The MP4(SDQ) procedure in G-16 is both economic and readily incorporated in subsequent processing to determine harmonic vibration frequencies. In the MOLPRO situation, wave-functions, determined from Møller-Plesset $4^{\text {th }}$ order perturbation theory, which included all single, double and quadruple substitutions (MP4(SDQ)) were structurally optimized by a combined iterative MP4SDQ and MCSCF procedure. Following a MP4(SDQ) all-electron run, the output wave-function was used as input to the MCSCF programme, where the 18 -valence electron closed shell MOs $\left(11 a^{\prime}+7 a^{\prime \prime}\right)$ were permuted into a sub-set of 13 active MOs $\left(7 a^{\prime}+6 a^{\prime \prime}\right)$. The gradients for the selected root (routine in MOLPRO) in the MCSCF generates the next geometry for input into the MP4(SDQ) run; the process is repeated until the gradients are lowered below the MOLPRO suite convergence limit.

A further important aspect, is that none of the low-lying TDDFT singlets and triplets which produced $\mathrm{C}_{2 \mathrm{~V}}$ upper states, did so under MP4(SDQ) conditions; the structures obtained showed that although the $\mathrm{C}_{1} \mathrm{C}_{2} \mathrm{C}_{3} \mathrm{C}_{4} \mathrm{C}_{5} \mathrm{C}_{6}$ unit was basically planar, the 7- $\mathrm{CH}_{2}$ group did not lie in the same plane and that one $\mathrm{H}$-atom lay almost directly above $\mathrm{C}_{7}$. Thus, the geometric differences were small but important, since they allowed the TDDFT wave-functions to be processed for vibrational composition. The corresponding vertically excited states were also obtained with the multi-reference, multi-root configuration interaction package (MRD-CI) ${ }^{34}$ within the GAMESS-UK method..$^{35}$

Franck-Condon (FC), Herzberg-Teller (HT) and combined (FCHT) vibrational analyses were performed on G-16 wave-functions using the Pisa software. ${ }^{36-38}$ When imaginary frequencies 
are present, our early version of the Pisa suite, can fail to identify the lower state mode which must be projected out, to equalise the mode numbers of upper and lower states. In those circumstances, the 39 vibrational modes for CHT can be processed as groups of active vibrations; the overall FC, FCHT or HT analysis is then built up by summation of the contributions from several computations. In HT calculations, although the structural symmetry of CHT is maintained, it is essential to remove all molecular symmetry from the wave-functions for both the upper and lower vibrational states to succeed; the transition dipole moment derivatives must also be stored on the checkpoint file.

All basis sets used were triple-zeta in the valence shell with polarization (TZVP), ${ }^{39-43}$ including aug-cc-pVTZ, ${ }^{39,40}$ the Pople style ${ }^{41} 6-311 \mathrm{G}^{* *}$ and Karlsruhe default $2^{\text {nd }}$ series with additional p- and d-functions (def2-TZVPPD); $;^{42,43}$ the choice varied with the numerical technique in progress. In vibrational analyses, the same basis set was used for upper and lower vibrational states.

Theoretical treatment of all Rydberg states, irrespective of s-, p-, d- or f- etc., requires the use of very diffuse atomic orbital (AOs) functions. As a result, the position of these within the molecular structure is relatively unimportant; here they were positioned on the $\mathrm{C}_{7}$-atom in order to retain molecular symmetry. In addition to the $6-311 \mathrm{G}^{* *}$ basis set, present on all atoms, the Gaussian type AOs orbitals with exponents $0.021,0.008,0.0025 .0 .0008$ and 0.00025 were used in GAMESS-UK. ${ }^{35}$ The values were chosen following our study of the VUV spectrum of cyclooctatetraene (COT) and much earlier work. ${ }^{2}$

2. The VUV spectrum. This was obtained in the gas-phase at room temperature, using the AU-UV beam line at the ASTRID2 synchrotron in Aarhus, Denmark. The procedure was similar to our previous VUV spectral studies, ${ }^{44}$ but further details are given in the supplementary material as SM1. The CHT sample, purchased from Sigma-Aldrich (C99205), was used without further purification. Our spectral range, $3.794 \mathrm{eV}\left(30600 \mathrm{~cm}^{-1}\right)$ to $10.79 \mathrm{eV}$ 
$\left(87029 \mathrm{~cm}^{-1}\right)$, was covered by 1859 data points with an increment of $0.05 \mathrm{~nm}$ above $148 \mathrm{~nm}$, and $0.1 \mathrm{~nm}$ below this wavelength. The monochromator spectral resolution is fixed at 0.075 $\mathrm{nm}$ across the full range and hence is non-linear in energy. Thus, the resolution changes from $0.85 \mathrm{meV}$ at $330 \mathrm{~nm}(3.756 \mathrm{eV})$, through $3.22 \mathrm{meV}$ at $170 \mathrm{~nm}(7.29 \mathrm{eV})$, to $6.45 \mathrm{meV}$ at 120 $\mathrm{nm}(10.32 \mathrm{eV})$. This leads to broader peaks being observed with increasing energy, even over relatively small energy differences. This is relevant to super-position of different spectral ranges as performed below; photons at 5 and $7 \mathrm{eV}$ have resolutions of 1.5 and $3 \mathrm{meV}$ respectively. Very weak structure in the spectrum was enhanced by fitting the underlying broad structure to a closely fitting polynomial function which best fits this local profile.

\section{RESULTS}

\section{A. Principal features of the experimental UV + VUV spectrum.}

The full VUV spectrum which presents mainly broad features is shown in Fig. 3; the wide scan theoretical set of singlet states superimposed, are discussed in Section 2 below, and listed in the supplementary material as SM2. Very weak fine structure is evident close to the minimum near $5.7 \mathrm{eV}$, as shown in Fig. 4. Subtraction of the fit from the observed signal and processing the regular residuals (RR) yields the result shown in blue in Fig. 4; this is also discussed further below.

Figure 3. The VUV spectrum with the lowest $150 \mathrm{VEE}$ singlet states (in blue) produced by the aug-cc-pVTZ basis set at the equilibrium structure of the $X^{1} A^{\prime}$ state. The oscillator strengths ( $f(r))$ for these were then replaced by Gaussian functions with the same amplitude but differing half widths at half-maximum (HWHM); these are shown in differing colors. 


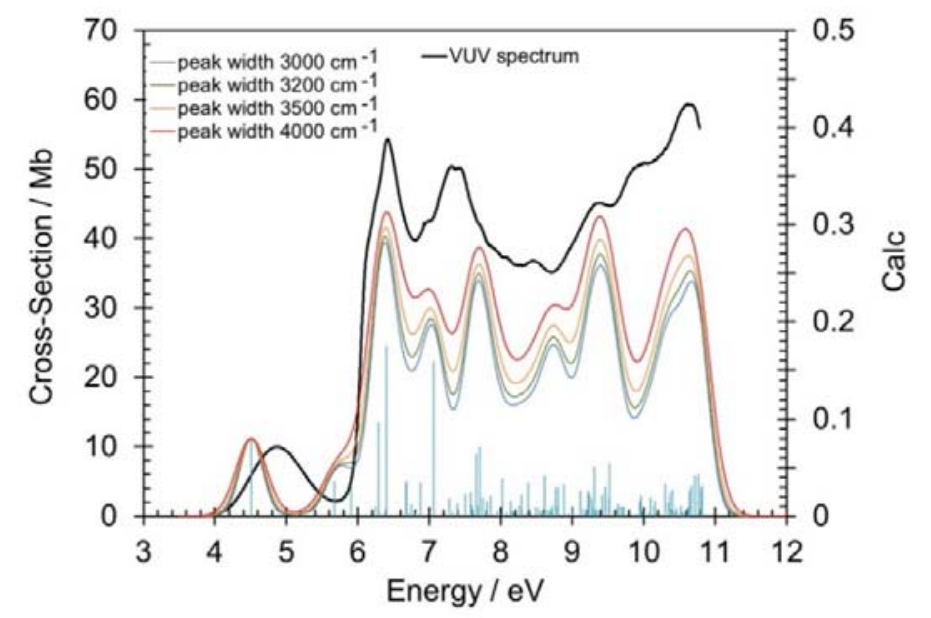

Figure 4. The VUV spectrum of CHT in the region between 5.2 and $6.0 \mathrm{eV}$ shows a set of very weak but sharp peaks close to the local minimum. After fitting to a local polynomial (shown in red), the regular residuals (RR) are plotted in blue. There are the same number of data points in the RR as the main spectrum, and these demonstrate the considerable detail present in the spectrum. The varying intensities probably indicate the presence of a complex vibrational pattern. The lines slightly broaden with increasing energy. There is no evidence of additional excited states contributing to the cross-section in this region, as discussed in the text.

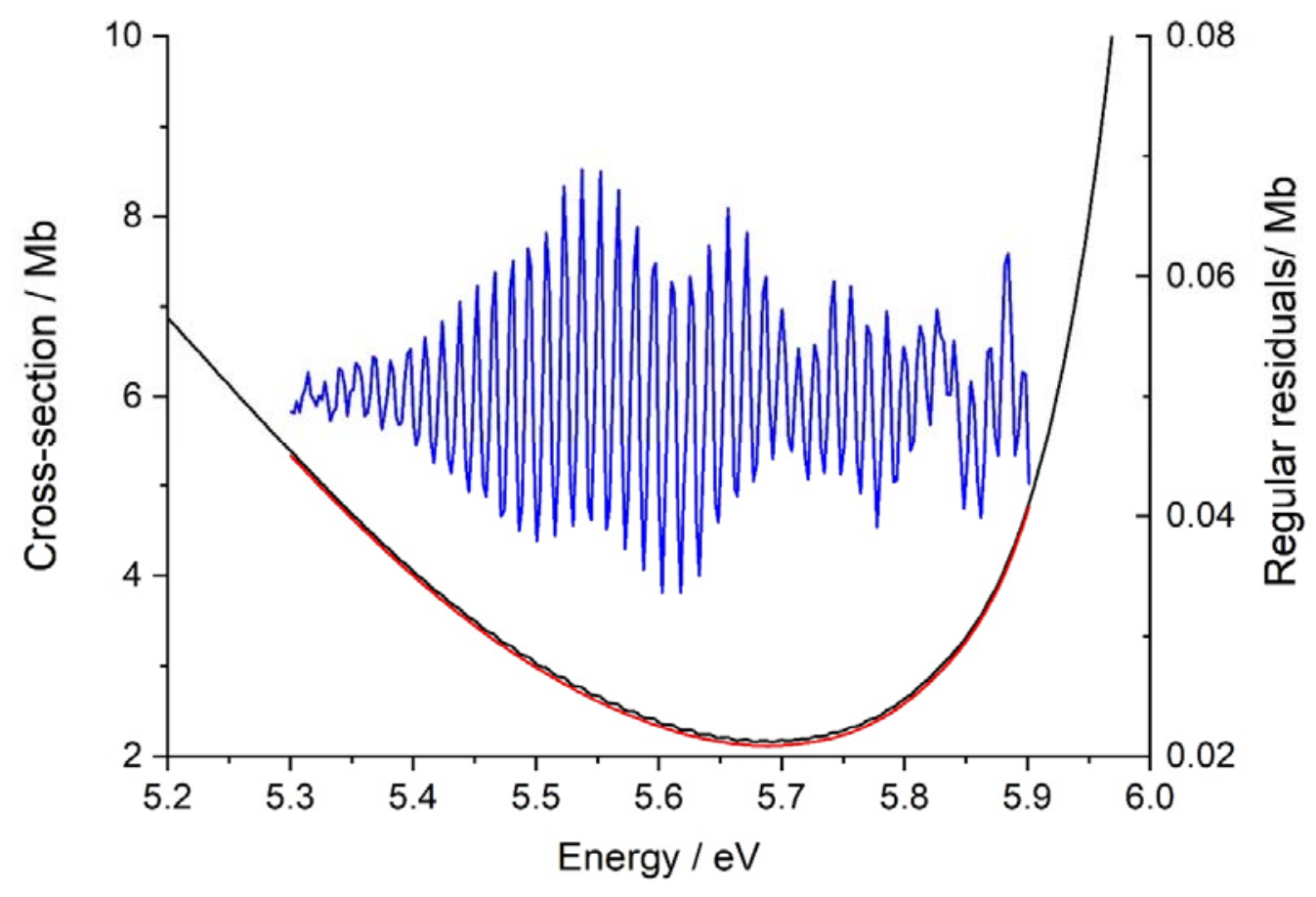


1. Overall interpretation of the spectral profile in the range up to $\mathbf{1 0 . 5} \mathrm{eV}$. The principal contributors to the spectral intensity are based on vertical excitation energies (VEE), using TDDFT together with the aug-cc-pVTZ basis set. A set of 150 VEE singlet states are shown as blue vertical bars alongside the VUV spectrum in Figure 3. In order to generate a theoretical profile, the vertical bars were replaced by Gaussian functions at the same energies. Varying line-widths, with half-width at half maximum (HWHM) in the range 3000 to $4000 \mathrm{~cm}^{-1}$ were used. Summation of the Gaussians showed that comparison of the theoretical and experimental envelopes, using a line-width of $3000 \mathrm{~cm}^{-1}$ gives the closest interpretation of the spectrum; this gives generally close agreement with the experimental profile, although some relatively minor differences are evident. We assign the strongest peaks in the VUV spectrum, based on these calculations, to the selected states in Table I. Those listed are the more dominant states, either from a distinct energy position or high intensity. The oscillator strengths (f(r)) vary widely. Most of the observed VUV maxima appear to be the summation of groups of electronic states, rather than individuals. Two theoretical states, $3^{1} \mathrm{~A}^{\prime}$ and $5^{1} \mathrm{~A}^{\prime}$, are dominant with high $\mathrm{f}(\mathrm{r})$; these have onsets separated by $\sim 1.0 \mathrm{eV}$. We correlate these with the major peaks centered on 6.41 and a doublet centered on $7.36 \mathrm{eV}$ in the VUV spectrum.

Recently, we interpreted the VUV spectrum of cyclooctatetraene similarly; ${ }^{45}$ there the unscaled VEE valence states, calculated by the MRD-CI method, gave a closet correlation with experiment when the FWHM $0.35 \mathrm{eV}\left(2823 \mathrm{~cm}^{-1}\right)$, similar to the present study. A more extensive list of excited states is shown in the supplementary material as SM2.

2. Comparison of the VUV spectrum with electron impact. The present UV+VUV absorption spectrum is compared in Fig. 5 with EI results from Knoop et al, ${ }^{25}$ who used a trapped electron low energy technique; this does not give similar spectral profiles to UV absorption. Knoop et $a^{25}$ assign triplet state maxima below $\mathrm{S}_{1}$ at 3.68 and $3.89 \mathrm{eV}$ to 
separate electronic states, but these could be vibrational structure from a single state. When CHT is diluted with helium, an even lower band close to $2.1 \mathrm{eV}$ was observed on the rapidly rising low energy wall; this was thought to be an excited state of an electron attached anion. $^{25}$

Frueholz et $a l^{26}$ used a conventional electron energy loss (EEL) technique; such spectra with impact energies in the range 30 to $75 \mathrm{eV}$, often give a close correspondence to VUV spectra; this appears here. The importance of the EEL spectra is that they enable us to see excitations below our lower energy limit for measurement at $3.794 \mathrm{eV}\left(30601 \mathrm{~cm}^{-1}\right)$, and also some triplet states. The most obvious similarities between the absorption and EI spectra occur in the 4 to 8 $\mathrm{eV}$ region. Numerical curve fitting to our spectrum, gives its maximum of $\mathrm{S}_{1}$ at 4.65 (1) $\mathrm{eV}$. The observation that this state rapidly undergoes internal conversion to a vibrationally excited version of the ground state $\left(\mathrm{S}_{0} *\right)$, was discussed above. ${ }^{15-22}$ However, none of these P-P studies, or the EI studies, reported the multiplet in the VUV minimum between 5.3 and $5.9 \mathrm{eV}$, possibly a result of the electron energy resolution of their experiments being lower than used here.

Figure 5. Comparison of the electron impact profiles given by Knoop et $\mathrm{al}^{25}$ (trapped electron) and Frueholz et al ${ }^{26}$ (EELS) with the present UV+VUV spectrum. The Frueholz et al ${ }^{26}$ spectra from the energy ranges in several Figures have been overlaid, leading to slight thickening of the combined lines. The similarity between the EELS and VUV spectra is as expected. 


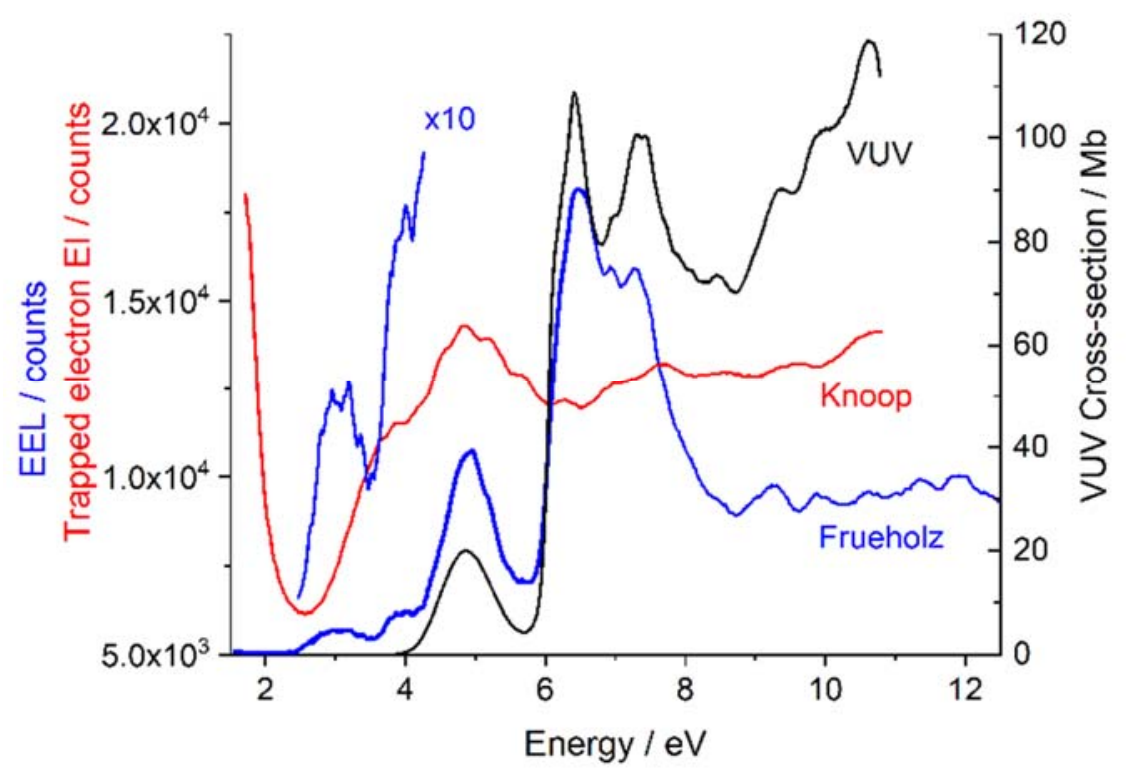

Comparing the two EI studies, ${ }^{25,26}$ there appear to be two experimental triplet states to assign. Assuming Cs symmetry, and at the MP4(SDQ) level of determination, our triplet lowest adiabatic excitation is $1^{3} \mathrm{~A}^{\prime \prime}\left(11 \mathrm{a}^{\prime} 8 \mathrm{a}^{\prime /}\right)$ at $2.290 \mathrm{eV}$. The $1^{3} \mathrm{~A}^{\prime}$ state $\left(7 \mathrm{a}^{\prime /} 8 \mathrm{a}^{\prime /}\right)$ is calculated to lie significantly higher in energy, at $4.286 \mathrm{eV}$. These two theoretical states are assigned to the EI vertical states at 3.05 and $3.95 \mathrm{eV}$. The next lowest, is a $\sigma \sigma^{*}$-triplet state at the MP4SDQ level; it is ${ }^{3} \mathrm{~A}^{\prime}\left(11 \mathrm{a}^{\prime} 12 \mathrm{a}^{\prime}\right)$ calculated to lie at $4.850 \mathrm{eV}$. When constrained to $\mathrm{C}_{2 \mathrm{~V}}$ symmetry, the lowest triplet states have AEE: $1.71\left(1^{3} \mathrm{~B}_{2}\right), 3.87\left(1^{3} \mathrm{~A}_{1}\right)$ and $5.13\left(2^{3} \mathrm{~B}_{2}\right)$, we believe that the Cs symmetry states represent reality better.

The lowest energy CHT anion, with ${ }^{2} \mathrm{~A}^{\prime \prime}$ symmetry $\left(8 \mathrm{a}^{\prime \prime}\right)$, is calculated to lie at $1.832 \mathrm{eV}$; this is relatively close to the anionic excited state at $2.1 \mathrm{eV}$ claimed by Knoop et al. ${ }^{26}$ The structures of all these species are shown in the Supplementary Material as SM3.

Table I. The most intense vertical singlet state excitation energies (VEE) for CHT using the aug-cc-pVTZ basis set with the B3LYP DFT functional. These are expected to strongly contribute to the intensity of the main peaks of the VUV spectrum.

\begin{tabular}{|c|c|c|c|c|c|}
\hline State & $\begin{array}{c}\text { Energy } \\
/ \mathrm{eV}\end{array}$ & $\begin{array}{c}\text { Oscillator } \\
\text { strength }\end{array}$ & State & $\begin{array}{c}\text { Energy } \\
/ \mathrm{eV}\end{array}$ & $\begin{array}{c}\text { Oscillator } \\
\text { strength }\end{array}$ \\
\hline $1 \mathrm{~A}^{\prime \prime}$ & 4.5086 & 0.0800 & $4 \mathrm{~A}^{\prime \prime}$ & 7.7045 & 0.0722 \\
\hline
\end{tabular}




\begin{tabular}{|c|c|c|c|c|c|}
\hline $1 \mathrm{~A}^{\prime}$ & 5.6745 & 0.0365 & $6 \mathrm{~A}^{\prime}$ & 8.0232 & 0.0391 \\
\hline $2 \mathrm{~A}^{\prime}$ & 6.2923 & 0.0971 & $7 \mathrm{~A}^{\prime}$ & 8.6158 & 0.0413 \\
\hline $3 \mathrm{~A}^{\prime}$ & 6.4025 & 0.1751 & $4 \mathrm{~A}^{\prime \prime}$ & 9.3100 & 0.0505 \\
\hline $4 \mathrm{~A}^{\prime}$ & 6.6802 & 0.0364 & $5 \mathrm{~A}^{\prime \prime}$ & 9.5209 & 0.0542 \\
\hline $5 \mathrm{~A}^{\prime}$ & 7.0587 & 0.1595 & $8 \mathrm{~A}^{\prime}$ & 10.7200 & 0.0420 \\
\hline $2 \mathrm{~A}^{\prime \prime}$ & 7.6542 & 0.0639 & $9 \mathrm{~A}^{\prime}$ & 10.7681 & 0.0430 \\
\hline
\end{tabular}

The present VEE calculations suggest that there are two valence states, at 5.67 and $5.91 \mathrm{eV}$, between the first two main bands of the VUV spectrum. This is the region where we have extracted the significant weak fine structure, shown in Figure 4. Although we believe this denotes the presence of a Rydberg state, we checked whether the undulation of the extracted fine structure profile represented further under-lying weak valence states. We sought these at a combined MP4(SDQ) with MCSCF level. The lowest AEE solution was a $1^{1} \mathrm{~A}^{\prime}$ doubly excited state at $4.579 \mathrm{eV}$ with process $15 \mathrm{a}^{\prime 2} \rightarrow 11 \mathrm{a}^{/ / 2}$; this doubly excited singlet state will not appear in the VUV absorption spectrum. This was followed by the $2^{1} \mathrm{~A}^{\prime \prime}$ state at $6.398 \mathrm{eV}\left(14 \mathrm{a}^{\prime}\right.$ $\left.\rightarrow 11 \mathrm{a}^{\prime /}\right)$. Neither of these can be responsible for the undulations observed in the 5.3 to $5.9 \mathrm{eV}$ region of the VUV, which must be elements of a vibrational envelope. The $\mathrm{S}_{1}$ onset of the $\mathrm{UV}+\mathrm{VUV}$ spectrum, $15 \mathrm{a}^{\prime} \rightarrow 11 \mathrm{a}^{\prime \prime}$, is also discussed in detail below.

Our singlet and triplet state adiabatic excitation energies (AEE), shown in Table II, were also computed using the TDDFT method. As implemented in G-16 and other suites, single excitation configuration interaction (CIS) methods such as TDDFT assign the ground state energy at the equilibrium structure of the excited state under investigation. This contrasts with spectroscopy, where AEE are defined as the energy difference between two states, each at their respective equilibrium structures. If there is little change in molecular structure between the upper and lower states, then the TDDFT definition will not be seriously in error. In flexible systems such as with CHT, this does not apply. In order to agree with the spectral AEE, the 'apparent' AEE of TDDFT require correction. In some cases, these corrections may be $1 \mathrm{eV}$ or more, and are shown in Table II, which shows both standard and 'corrected' values. 
Only the lowest few AEE were obtained, since attempts to generate higher states often led to collapse of the wave-function to a lower state of same symmetry. For example, when seeking to optimize the $9^{\text {th }}$ root in the B3LYP sequence using G-16, and starting at the $\mathrm{X}^{1} \mathrm{~A}^{\prime}$ structure, the energy is $8.430 \mathrm{eV}$. This state then partially optimizes through an $\mathrm{A}^{\prime}$ state with energy $6.779 \mathrm{eV}$ having very high oscillator strength $(0.7742)$; however, it finally converges, in energy and oscillator strength, to the same state as the $3^{\text {rd }}$ root shown in Table II. Consequently, vertical studies described here, and shown in Figure 3, became important.

Table II. The adiabatic excitation energies (AEE) for low-lying singlet valence states of CHT. These were determined using the TDDFT/ CIS method with the B3LYP functional. The apparent 'AEE' shown are defined as the difference in energies of the ground and excited states at the equilibrium structure of the excited state. In spectroscopy both these state energies are defined at their respective equilibrium structures; these are shown as AEE corrected. The canonical sequence of MOs involved is also shown.

\begin{tabular}{|c|c|c|c|c|}
\hline $\begin{array}{c}\text { G-16 } \\
\text { 'AEE' } \\
/ \mathrm{eV}\end{array}$ & $\begin{array}{c}\text { AEE } \\
\text { corrected } \\
/ \mathrm{eV}\end{array}$ & Symmetry & $\begin{array}{c}\text { Oscillator } \\
\text { strength }\end{array}$ & $\begin{array}{c}\text { Open shell leading terms } \\
\text { Eigenvector and SCF MO sequence. }\end{array}$ \\
\hline 3.117 & 4.527 & $1^{1} \mathrm{~A}^{\prime \prime}$ & 0.0986 & $0.6924(25-26)$ \\
\hline 4.066 & 4.600 & $1^{1} \mathrm{~A}^{\prime}$ & 0.0117 & $0.6269(24-26) \mathrm{a}^{\prime /} \mathrm{a}^{\prime \prime}+0.3244(25-27)$ \\
\hline 5.68 & 6.731 & $2^{1} \mathrm{~A}^{\prime}$ & 0.3280 & $(25-27) \mathrm{a}^{\prime} \mathrm{a}^{\prime}-(24-26) \mathrm{a}^{\prime /} \mathrm{a}^{\prime /}$ \\
\hline 5.930 & 6.408 & $2^{1} \mathrm{~A}^{\prime \prime}$ & 0.0328 & $0.4312(24-27) \mathrm{a}^{\prime} \mathrm{a}^{\prime /}+0.4040(23-26) \mathrm{a}^{\prime \prime} \mathrm{a}^{\prime}$ \\
\hline 6.462 & 7.146 & $3^{1} \mathrm{~A}^{\prime \prime}$ & 0.1951 & $(24-27) \mathrm{a}^{\prime /} \mathrm{a}^{\prime}+(25-26) \mathrm{a}^{\prime} \mathrm{a}^{\prime /}$ \\
\hline
\end{tabular}

3. Vibrational structure in the experimental UV + VUV spectrum. (a) Predictive assignment for the Rydberg states in the VUV spectrum. Since the photoelectron spectrum of CHT does not show vibrational structure on the lowest ionic state, ${ }^{1}$ it is necessary to evaluate Rydberg state energies based upon the VIE rather than AIE; the two lowest VIE are 8.55(1) and 9.49(1) eV. The regular expression relating the ionization energy (IE), Rydberg state energy (E), its principal quantum number $(n)$ and its quantum defect $(\delta)$ is shown in Equation 1.

$$
\text { IE }-E_{n}=13.61 /(n-\delta)^{2} \quad \text { Equation } 1
$$


For Rydberg states, the normal range for $n s$-states is $0.8 \leq \delta \leq 1.0$; for $n p$-states $0.3 \leq \delta \leq$ 0.6 and for $n d$-states $0.0 \leq \delta \leq 0.2$. The $n s$-Rydberg states having $n=3,4$ and 5 , with an assumed value for $\delta=0.9$, have predicted energies $5.464,7.134$ and $7.740 \mathrm{eV}$; the first is close to the vibrational sequence between 5.3 and $5.9 \mathrm{eV}$; the predicted higher values assist the search for the observed states. Since many Rydberg states, irrespective of $n$ and $\delta$, show similar vibrational patterns, we seek groups of lines at higher energies exhibiting complex vibrational profiles, while remembering that the individual s-, p-, d- states etc. will become progressively closer, and partially overlay one another.

We performed a series of polynomial 'best fits' to the data points of several local energy ranges; the best fit cross-sections after subtraction from the VUV total spectral cross-section data, yield the regular residuals (RR),are displayed in Figure 6. The exponential character of the series energies is apparent from the 'predicted' positions for several low-lying Rydberg states shown. Rydberg states were established by super-position of portions of the VUV spectrum, after an energy shift of one or other state to higher or lower energy. This enables relative energies of the two states to be established.

Figure 6. The regular residuals from the VUV spectrum with predicted values for s-, pand d-Rydberg states; values of the principal quantum numbers $(n)$ are from 3 to 11, using typical delta values as discussed in the text. Since $p$-Rydberg states can show a wide range of delta values, two values are shown. A number of relatively high cross-section peaks occur which are at significantly different energies from the predicted Rydberg 
states.

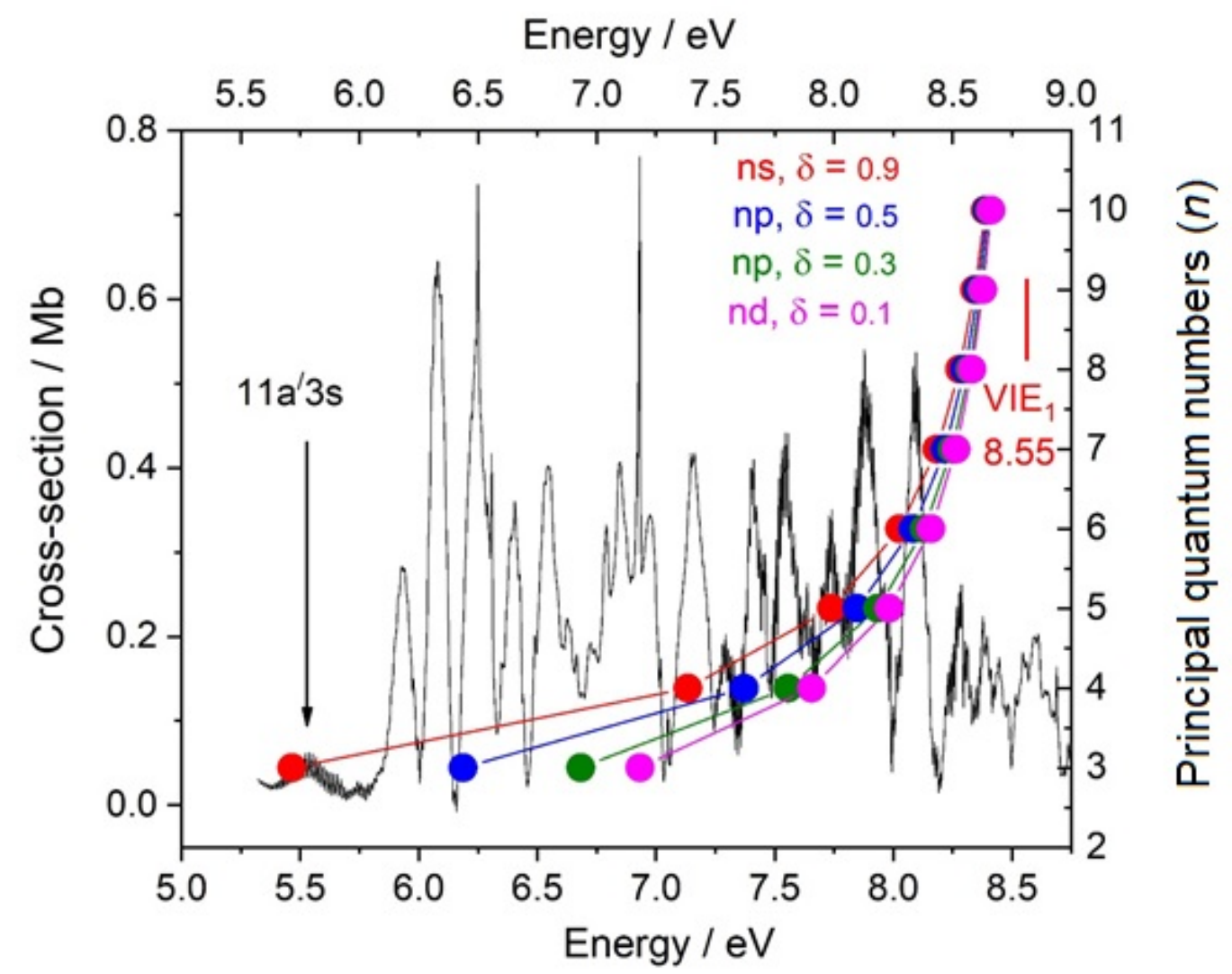

(b) The region from 5.3 to $5.9 \mathrm{eV}$. This region between the first and second main absorption bands of the spectrum in Figure 4, shows a long vibrational series of 33 or more maxima, with slight broadening towards higher energies. The lack of apparent anharmonicity is surprising but is not unique; ${ }^{45}$ for example, the trans-but-2-ene cation shows 'remarkable harmonicity' in its low frequency modes; this contrasts with the corresponding propene ionic state. ${ }^{45}$ Since both of these hydrocarbons are exhibiting $\pi$-ionizations, the overall situation is complex. The apparent onset is close to $5.15 \mathrm{eV}\left(43000 \mathrm{~cm}^{-1}\right)$, while the median separation of the peaks, 115 $\mathrm{cm}^{-1}$, has a very low standard deviation (SD) of $5.5 \mathrm{~cm}^{-1}$. We conclude that this group of lines show the presence of a Rydberg state with variable intensities, and slightly increasing linewidth with increasing energy. No electronic states with an AEE in this energy range were found at the MP4(SDQ)+MCSCF level described above; this appears to exclude an underlying very weak valence state in the region, confirming the presence of a Rydberg state. 
Several higher energy groups of vibrational states, apparent in Figure 6, are also thought to be Rydberg states. These spectral groups are not expected to show identical multiplets; broadening from the spectrometer, and overlay of states with differing spherical harmonics, will destroy part of the structure. As an example, we compare the 5.3 to $5.9 \mathrm{eV}$ multiplet with the spectrum at $7.737 \mathrm{eV}$ in Figure 7, where similar structure occurs. RR from the $5.535 \mathrm{eV}$, lower energy state, are shown in blue, with those shifted from a higher region of the VUV in red. As mentioned in Section 2 on Methods above, the difference in resolution for the two spectral regions is very apparent; however, there is a close correspondence between the red and blue profiles, although some mismatches occur. We conclude that there is enough similarity between the two red and one blue lines, to confirm the suggestion that both are Rydberg states. The mismatches probably arise from either interference with valence structure underlying one or other spectrum, or the presence of other Rydberg states in the same energy region. In more detail, the red superimposed spectrum in Figure 7 shows four groups of lines with centroids having approximate separations of 2903, 1480 and $928 \mathrm{~cm}^{-1}$; these are consistent with vibrational separations, but the separations are too small to be separate Rydberg states.

Figure 7. The $5.5 \mathrm{eV}$ region of the VUV spectrum (in blue); superposition of the regular residuals from the $7.737 \mathrm{eV}$ region of the VUV spectrum (in red). The two spectra are aligned at $5.535 \mathrm{eV}$ after reducing the red spectral energy by $2.200 \mathrm{eV}$. Many of the peaks superimpose for both VUV bands, showing that both contain a Rydberg state. However, further from the correlation energy used here, mismatches occur, possibly a result of perturbation by one or more states on either reference state. 


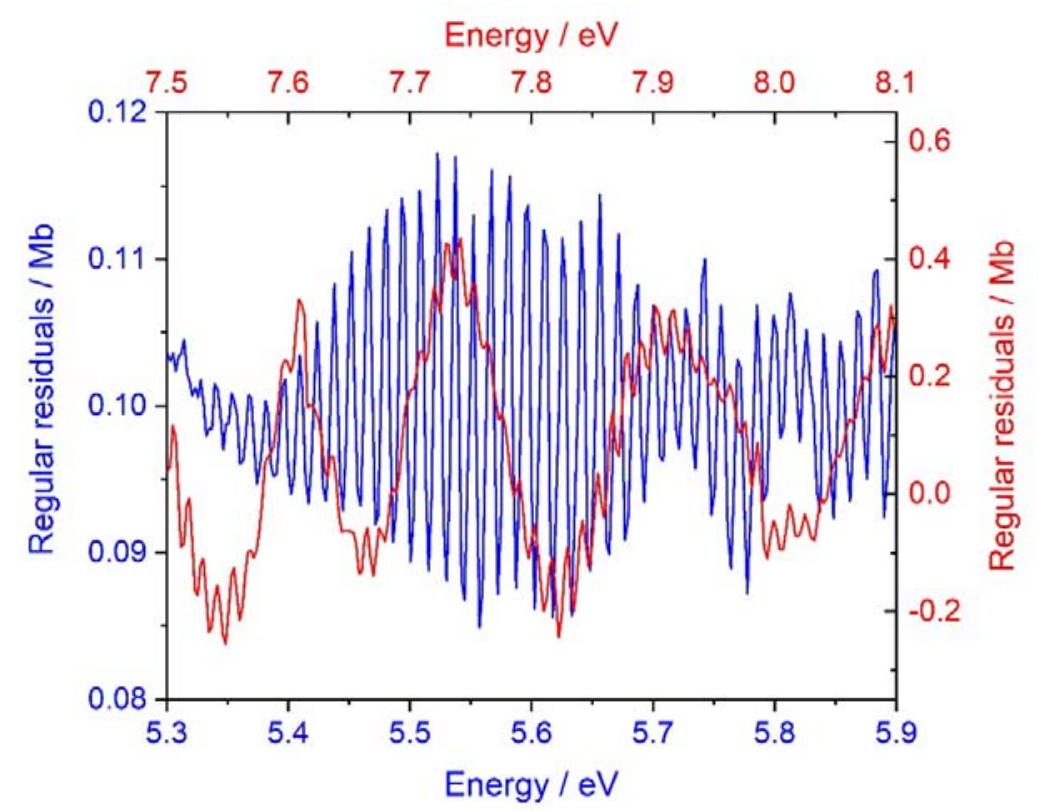

Insertion of the $7.737 \mathrm{eV}$ VUV band energy into Equation 1 above with the experimental VIE of $8.55(1) \mathrm{eV}$, leads to $n=5$ with quantum defect $(\delta) 0.908$, and hence identification as a $5 \mathrm{~s}-$ Rydberg state. The same procedure when applied to the leading maximum of the lower energy state (blue line) with local maximum at $5.535 \mathrm{eV}$, gives $n=3$ with $\delta=0.875$; this is the $3 \mathrm{~s}$ Rydberg state. Both are based on excitations from the $11 \mathrm{a}^{\prime} \mathrm{HOMO}$.

(c) Rydberg states in the VUV spectrum above $7 \mathrm{eV}$. The regular residuals (RR) of higher energy portions of the spectrum, after removal of local broad structure via subtraction of fitted polynomial functions, show a considerable number of sharp peaks; these are depicted in Fig. 8. It is important to note that the subtraction process generates the same number of RR data points as in the original spectrum; this increases the RR significance owing to the considerable number of data points (10 in most cases) under each peak. In principle, some of these RR peaks could be remnants from valence states, since much of the intensity has been removed by the subtraction process; however, such remnants are unlikely to show this sharp structure. In contrast, Rydberg states normally have sharp but weak peaks, with low oscillator strength, matching the situation here. These can easily be missed when superimposed on strong 
background absorption; this loss of detail is unlikely when the subtraction procedure is used. The position of the 3p-Rydberg state is predicted in Figure 6 to lie between 6.4 and $6.7 \mathrm{eV}$, depending on the quantum defect $(\delta)$. The spectral peak at $6.50 \mathrm{eV}$ is broader than its neighbours, and all are clearly valence in nature with no evidence of fine structure, therefore it is not possible to definitively make an assignment of the 3p-Rydberg state. The most obvious presence of Rydberg states which lie higher than $7.25 \mathrm{eV}$, are shown in Figure 8. The energy range is limited to $\mathrm{VIE}_{1}$ at $8.55 \mathrm{eV}$, but a wider energy range is shown in the supplementary material as SM4.

Figure 8. The regular residual spectrum after removal of broad structure in the energy range 7.2 to $8.55 \mathrm{eV}$ (the lowest VIE). This shows a high vibrational density of absorption near $5.5 \mathrm{eV}$ (see Figure 4) attributed to Rydberg states, and especially above 7.25 eV. The spectrum contains the same number of data points as the original spectrum prior to the fitting process.

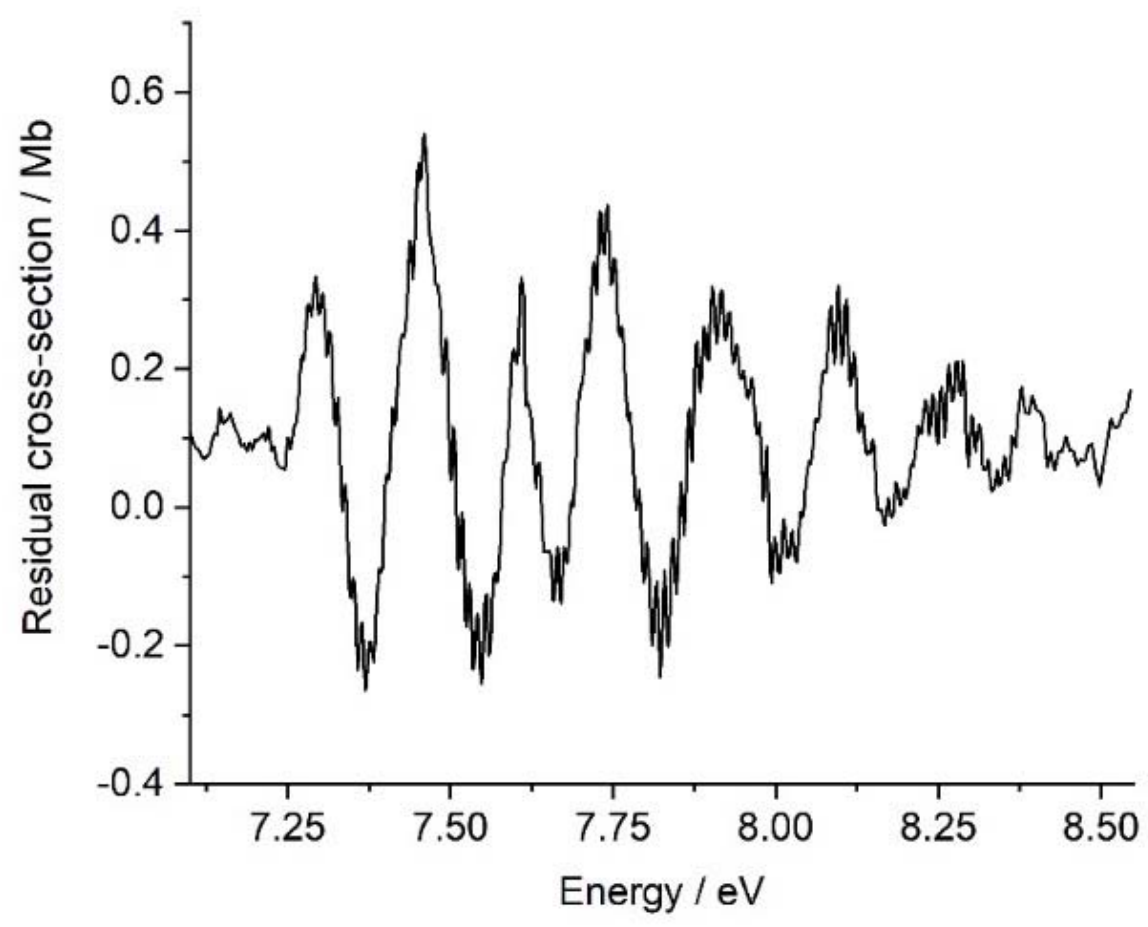

A further application of our overlay procedure, this time to a higher region of the VUV spectrum, is shown in Figure 9. There are a considerable number of peak super-positions, but 
as in Figure 7, mis-matches also occur. The red line at $8.111 \mathrm{eV}$, lowered by $2.560 \mathrm{eV}$ for coincidence with the blue line, gives a p-Rydberg state with $n=6$, with $\delta=0.432$, respectively. Although all the energies are only approximate, being based upon the visually assessed centroids of the bands, we believe that nine Rydberg states can provisionally be assigned in the VUV spectrum up to VIE1. Their energies, together with derived $n$ and $\delta$, using Equation 1, are as follows: $5.54(n=3, \delta 0.875), 7.29(4,0.711), 7.45(4,0.478), 7.61(4,0.197), 7.74(5,0.908)$, $7.91(5,0.396), 8.11(6,0.432), 8.24(7,0.353), 8.28 \mathrm{eV}(8,0.887)$. These three sets of values generate 27 items of data and allow a multivariate analysis ${ }^{46}$ in a non-linear least squares fitting program (Gnuplot), seeking the vertical ionization energy (VIE1) in Equation 1. A least-squares fit of these nine sets of measured data to Equation 1 above, for Rydberg state energies, gives the VIE 1 as $8.675 \pm 0.077 \mathrm{eV}$ with a low fit error of $0.88 \%$. This is in relatively close agreement with the experimental $\mathrm{VIE}_{1}$ of $8.55(1) \mathrm{eV}$, and cannot be a simple coincidence, given the range that Equation 1 could produce.

Figure 9. The regular residuals from a band at $8.111 \mathrm{eV}$, after reducing its energy by $2.560 \mathrm{eV}$, to super-impose the $5.535 \mathrm{eV}$ region of the VUV spectrum. Many of the peaks superimpose the 8.10 eV VUV band, showing that both contain the same Rydberg state. 


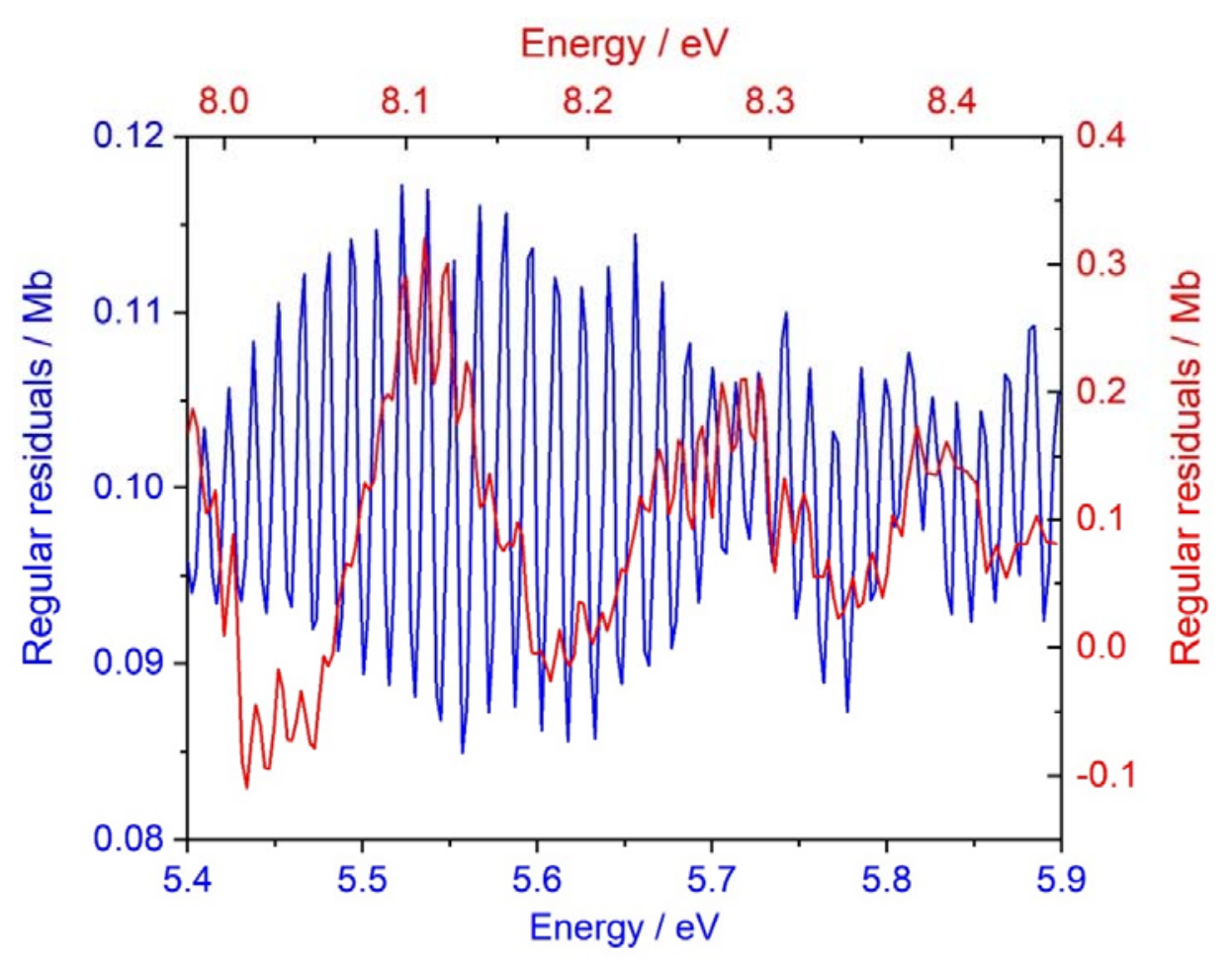

B. Theoretical study of the CHT excited states. 1.Vertical excitation energies (VEE)

for the valence states. Some of these were shown in Table I above. A more complete list is shown in the supplementary material as SM2. The most important MOs which occur in the lower excited states where Cs symmetry prevails, are shown in Fig. 10; the iso-electron density contours are at $0.02 \mathrm{e}$. The lowest pair of virtual MOs (VMOs) 26* and $27^{*}$ are strongly implicated in the UV and VUV spectra. VMO $26^{*}$ undergoes modification to a $\mathrm{C}_{2} \mathrm{~V}$ planar form $\left(\mathrm{a}_{2}{ }^{*}\right)$ in some TDDFT calculations via $\mathrm{CS}_{\mathrm{S}} \rightarrow \mathrm{C}_{2 \mathrm{~V}}$ conversion. However, this change of symmetry is not supported by the more sophisticated calculations at the MP4(SDQ) + MCSCF level above, which were generated using MOLPRO; we do not have access to a package to introduce MOLPRO wave-functions into G-16. However, since the structural differences between the $\mathrm{CS}_{\mathrm{S}}$ (MOLPRO) and $\mathrm{C}_{2 \mathrm{~V}}$ (G-16) structures are very small, the vibrational analysis using the $\mathrm{C}_{2 \mathrm{~V}}$ structures within the G-16 Pisa software became possible. 
Figure 10. The orientation of the four highest occupied and two lowest unoccupied MOs of CHT. The iso-electron density contours $(0.02 e)$ show a complex set of atomic orbital (AO) interactions. The lowest pair of virtual MOs 26* and 27*are strongly implicated in the UV and vacuum ultraviolet (VUV) spectra; these show nodal properties like the degenerate $1 \mathrm{e}_{2 u}{ }^{*}$ pair in benzene.

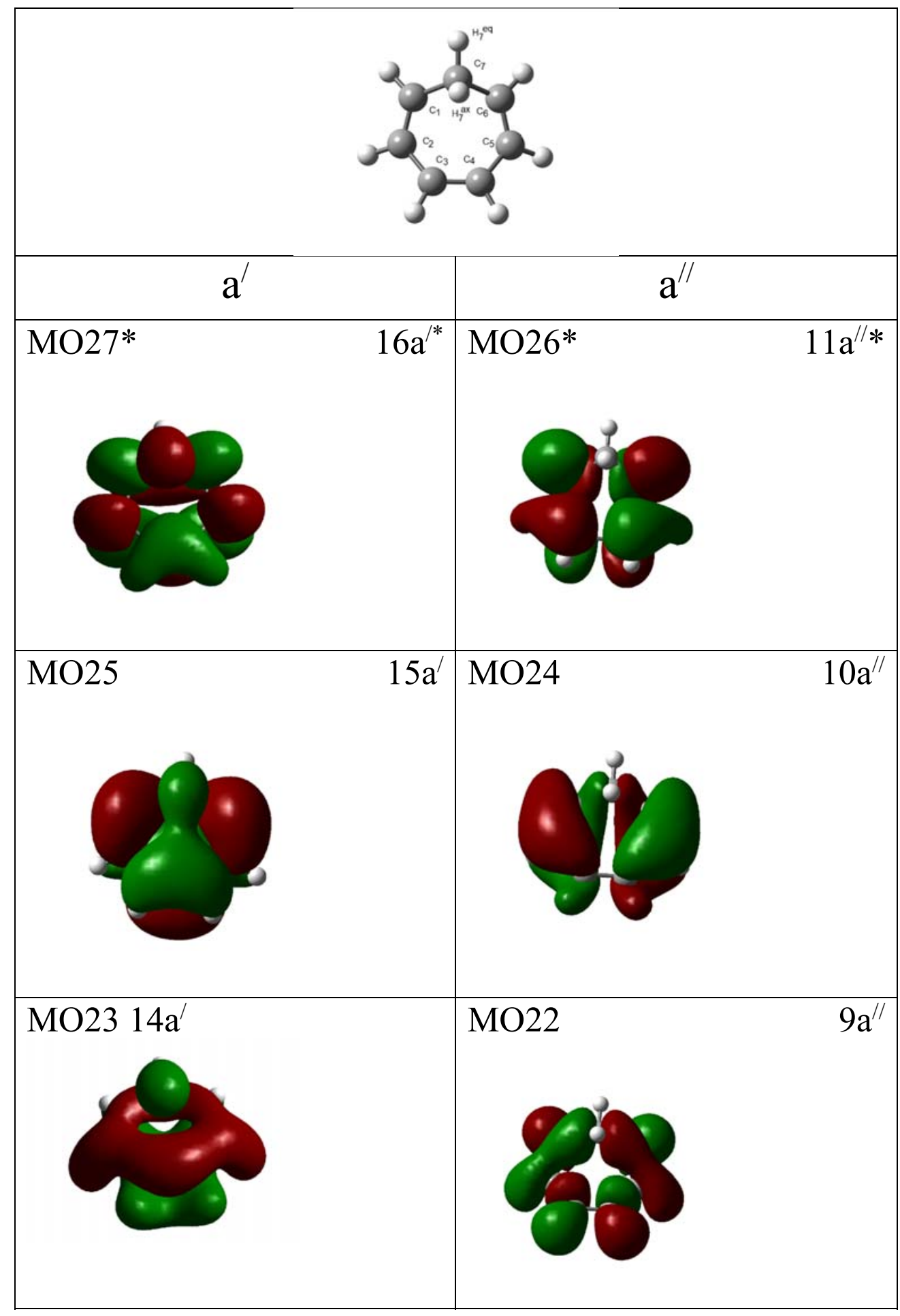


2. Theoretical Rydberg states. The Rydberg atomic orbitals (AOs) were solely positioned on the $\mathrm{C}_{7}$-atom, in order to preserve $\mathrm{Cs}_{\text {s symmetry. Corrected vertical excited }}$ state energies and their intensities were determined using TDDFT. The results for the lowest excitations to $3 \mathrm{~s}$, $4 \mathrm{~s}$ and $5 \mathrm{~s}$-Rydberg states are shown in Table III, where valence states relating to the $6-311 \mathrm{G}^{* *}$ basis set, produced alongside the Rydberg states (RS), have been omitted. The RS energies were extrapolated using Equation 1, as above with the experimental data, yielding the AIE, in Equation 1: $7.837 \mathrm{eV}$ (5) and $\delta 0.964$ (2), with fit errors in parentheses. The AIE (onset) of the PES spectrum is probably below $8.00 \mathrm{eV},{ }^{1}$ with its maximum (VIE) near $8.55(1) \mathrm{eV}$; the extrapolated theoretical results are in satisfactory agreement with that view. As expected, these projections leading to the AIE, are lower than the VIE extrapolated experimental results in Section 3(c) above.

An overall scan of the theoretical spectrum when s-, p- or d-Rydberg functions are present at the vertical excitation level, is shown in the supplementary material as SM5. This shows that most valence state energies and intensities are virtually unaffected by the presence of Rydberg AOs.

Table III. Adiabatic excitation energies (AEE), oscillator strengths, for singlet states when Rydberg basis functions are included to generate Rydberg states.

\begin{tabular}{|c|c|c|c|c|c|c|}
\hline $\begin{array}{c}\text { 'AEE' } \\
\text { /eV }\end{array}$ & $\begin{array}{c}\text { Corrected } \\
\text { AEE } / \mathrm{eV}\end{array}$ & State & $\delta$ & Symmetry & $\begin{array}{c}\text { Oscillator } \\
\text { strength }\end{array}$ & $\begin{array}{c}\text { Wave-function } \\
\text { leading terms }\end{array}$ \\
\hline 4.1317 & 4.5565 & $3 \mathrm{~s}$ & 0.963 & ${ }^{1} \mathrm{~A}^{\prime}$ & 0.0001 & $11 \mathrm{a}^{\prime} 12 \mathrm{a}^{\prime *}$ \\
\hline 5.3405 & 6.3580 & $4 \mathrm{~s}$ & 0.966 & ${ }^{1} \mathrm{~A}^{\prime}$ & 0.0001 & $11 \mathrm{a}^{\prime} 13 \mathrm{a}^{*}$ \\
\hline 5.9467 & 7.0041 & $5 \mathrm{~s}$ & 0.958 & ${ }^{1} \mathrm{~A}^{\prime}$ & 0.0001 & $11 \mathrm{a}^{\prime} 14 \mathrm{a}^{\prime *}$ \\
\hline
\end{tabular}

3. Vibrational analysis of the $3 s-R y d b e r g$ state via the $\mathbf{X}^{2} \mathbf{A}^{\prime}$ ionic state. The $3 \mathrm{~s}$ Rydberg state is shown in blue in Figures 4, 7 and 9. Here we extend our previous 
detailed theoretical analysis of the $\mathrm{VIE}_{1}$ state at the MP4(SDQ) level ${ }^{1}$ these calculations are simpler to perform than the corresponding Rydberg state ones, owing to the absence of very diffuse AOs, and some of the numerical instability that they can introduce. Using standard spectroscopic notation, where the highest fundamental vibration frequency is mode 1 , the lowest frequency vibration is mode $21\left(21 a^{\prime}\right)$. The theoretical PES spectrum was characterized by many quanta of mode 21, both as a fundamental and in binary and higher vibrational modes. The full-width of $\mathrm{IE}_{1}$ is over $0.9 \mathrm{eV}\left(\sim 7500 \mathrm{~cm}^{-1}\right)$, in comparison with the Rydberg state range observed in the VUV $(\sim 0.6 \mathrm{eV})$. The calculated ${ }^{2} \mathrm{~A}^{\prime}$ ionic state harmonic frequencies, superimposed on the PES, ${ }^{1}$ show that hot bands have a marked effect on both the width and complexity of the calculated PES spectrum, and we expect that the same will apply to the VUV spectral band.

Visually, the observed Rydberg state and calculated ${ }^{2} \mathrm{~A}^{\prime}$ state profiles in Figure 11 are sufficiently similar, that we regard the common identity to the Rydberg state and its limiting IE as secure. Clearly, mismatches occur, and the calculated PES and experimental VUV envelopes are not exact. The 3s-Rydberg state at $115(6) \mathrm{cm}^{-1}$, is some $25 \%$ higher. It is often found that very low frequency modes are calculated too low in energy; in this instance even the MP4(SDQ) level seems inadequate.

We have pursued our analysis to the anharmonic frequency level, as a potential solution to the low mode 21 frequency. The symmetric (a/) modes for both the harmonic (HAR) and anharmonic (ANHAR) frequencies for CHT are shown in Table IV; the lowest group of FC contributions to the $\mathrm{X}^{2} \mathrm{~A}^{\prime}$ ionic state are shown in Table $\mathrm{V}$. In both series, this mode is dominant, with 10 quanta of this mode occurring as single vibrational states in Table V. However, the frequency differences between HAR and ANHAR are relatively small, and mode 21 is certainly not raised in magnitude. 
The theoretical onset of the $\mathrm{X}^{2} \mathrm{~A}^{\prime}$ ionic state anharmonic frequencies is shown in Figure 12 . Band broadening has been simulated by means of Gaussian functions with Half-Widths at Half-Maximum (HWHM) of $10 \mathrm{~cm}^{-1}$, shown in red. The bands are basically doublets, where the intensity passes from the lower wavenumber series to a slightly higher wavenumber series. When the linewidth is increased to $20 \mathrm{~cm}^{-1}$, shown in blue, these doublets are accumulated into single peaks. This is lower than the peak width of the VUV spectrum, and the result is more similar to the observed 5.3 to $5.9 \mathrm{eV}$ band of the VUV spectrum. Although from a very different excitation, the synchrotron based VUV multiphoton excitation spectrum of the $\mathrm{X}^{2} \mathrm{~A}_{2}$ " state of the ammonia cation shows a very similar profile where intensity passes from one multiplet to another. ${ }^{47}$

Overall, we believe that the apparently monotonic progression observed with frequency 115 $\mathrm{cm}^{-1}$, and SD of only $5.5 \mathrm{~cm}^{-1}$, is accidental. Clearly a variety of $\mathrm{a}^{\prime}$ modes are involved both as fundamentals and combinations, as shown in Table V. The appearance of the onset can be significantly changed by small changes of the very narrow line-width.

Thus, a higher level theoretical treatment is needed for accurate reproduction of the experimental Rydberg state; this is beyond the scope of the present study. However, we can project the results for the ${ }^{2} \mathrm{~A}^{\prime}$ state and directly to the Rydberg states, backwards for this phenomenon. We have found that less sophisticated methods than the current ones, such as single excitation CI within TDDFT, is even poorer than the MP4(SDQ) and give negative frequencies for this mode in our hands.

Figure 11. The VUV 3s-Rydberg state, with separate hot (red) and cold band (blue) profiles from the MP4(SDQ) $\mathrm{X}^{2} \mathrm{~A}^{\prime}$ state. In order to reduce the congestion, the hot and cold bands have had their relative intensities adjusted from those reported previously. ${ }^{1}$ The calculated frequencies for the ionic state are not identical to those observed experimentally for the VUV state. This is especially true for the lowest frequency, where the calculated MP4(SDQ) value is $88 \mathrm{~cm}^{-1}$ to be compared with the observed line separation $115 \mathrm{~cm}^{-1}$; less sophisticated methods such as TDDFT give negative frequencies. 


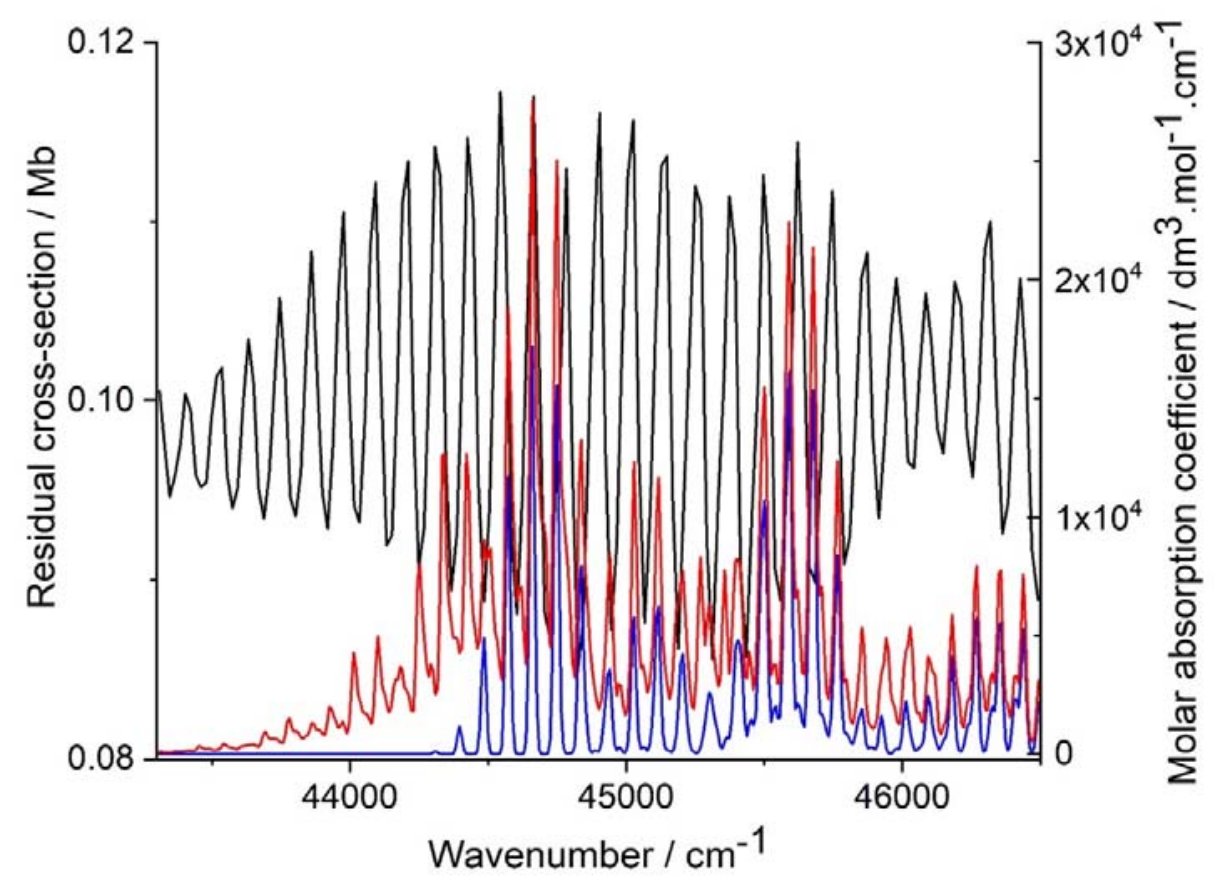

Table IV. The $\mathrm{X}^{1} \mathrm{~A}^{\prime}$ and ${ }^{2} \mathrm{~A}^{\prime}$ state $\mathrm{a}^{\prime}$ modes used in the Franck-Condon analyses of the $\mathrm{X}^{2} \mathrm{~A}^{\prime}$ ionic state and applied to the 3s-Rydberg state. The full list is given in the supplementary material.

\begin{tabular}{|l|l|l|l|l|l|l|}
\hline \multicolumn{3}{|c|}{$\mathbf{X}^{\mathbf{1}} \mathbf{A}^{\prime}$ state } & \multicolumn{3}{c|}{${ }^{\mathbf{2}} \mathbf{A}^{\prime}$ state } \\
\hline Mode & $\begin{array}{l}\text { Harmonic } \\
\text { Frequency } \\
/ \mathrm{cm}^{-1}\end{array}$ & $\begin{array}{l}\text { Anharmonic } \\
\text { Frequency } \\
/ \mathrm{cm}^{-1}\end{array}$ & $\begin{array}{l}\text { Difference } \\
/ \mathrm{cm}^{-1}\end{array}$ & $\begin{array}{l}\text { Harmonic } \\
\text { Frequency } \\
/ \mathrm{cm}^{-1}\end{array}$ & $\begin{array}{l}\text { Anharmonic } \\
\text { Frequency } \\
/ \mathrm{cm}^{-1}\end{array}$ & $\begin{array}{l}\text { Difference } \\
/ \mathrm{cm}^{-1}\end{array}$ \\
\hline 1 & 3160 & 3023 & -137 & 3240 & 3096 & -144 \\
\hline 2 & 3149 & 3013 & -136 & 3227 & 3036 & -192 \\
\hline 3 & 3128 & 2945 & -183 & 3217 & 3001 & -216 \\
\hline 4 & 3086 & 2955 & -131 & 3141 & 2746 & -395 \\
\hline 5 & 2991 & 2819 & -172 & 2940 & 2753 & -187 \\
\hline 6 & 1671 & 1635 & -35 & 1671 & 1605 & -67 \\
\hline 7 & 1572 & 1527 & -45 & 1608 & 1550 & -58 \\
\hline 8 & 1481 & 1436 & -45 & 1495 & 1451 & -44 \\
\hline 9 & 1426 & 1395 & -31 & 1442 & 1378 & -63 \\
\hline 10 & 1248 & 1231 & -17 & 1318 & 1299 & -20 \\
\hline 11 & 1222 & 1204 & -18 & 1265 & 1229 & -36 \\
\hline 12 & 1041 & 1018 & -22 & 1092 & 1025 & -67 \\
\hline 13 & 977 & 961 & -17 & 977 & 939 & -38 \\
\hline 14 & 939 & 921 & -18 & 959 & 929 & -31 \\
\hline 15 & 921 & 904 & -17 & 933 & 881 & -52 \\
\hline 16 & 812 & 802 & -10 & 830 & 791 & -39 \\
\hline 17 & 726 & 712 & -13 & 792 & 782 & -10 \\
\hline 18 & 668 & 661 & -7 & 643 & 616 & -27 \\
\hline 19 & 432 & 429 & -3 & 404 & 406 & 2 \\
\hline 20 & 356 & 353 & -4 & 367 & 355 & -12 \\
\hline 21 & 214 & 209 & -5 & 88 & 85 & -2 \\
\hline & & & & & & \\
\hline
\end{tabular}


Table V. The lowest vibrational states for the $\mathrm{X}^{2} \mathrm{~A}^{\prime}$ cation which are prominent in the 3sRydberg state analysis. Most are combinations of several quanta of mode 21 together with modes $20,19,18,17,16$ and 15.

\begin{tabular}{|l|l|l|}
\hline Vibrational state $/ \mathrm{cm}^{-1}$ & Mode and quanta & Intensity \\
\hline 0 & 0 & 123 \\
\hline 85 & $21^{1}$ & 790 \\
\hline 170 & $21^{2}$ & 3477 \\
\hline 255 & $21^{3}$ & 8733 \\
\hline 340 & $21^{4}$ & 13520 \\
\hline 425 & $21^{5}$ & 12820 \\
\hline 440 & $20^{1} ; 21^{1}$ & 208 \\
\hline 510 & $21^{6}$ & 6621 \\
\hline 525 & $20^{1} ; 21^{2}$ & 995 \\
\hline 595 & $21^{7}$ & 1134 \\
\hline 610 & $20^{1} ; 21^{3}$ & 2746 \\
\hline 661 & $19^{1} ; 21^{3}$ & 299 \\
\hline 695 & $20^{1} ; 21^{4}$ & 4751 \\
\hline 746 & $19^{1} ; 21^{4}$ & 322 \\
\hline 765 & $21^{9}$ & 751 \\
\hline 780 & $20^{1} ; 21^{5}$ & 5178 \\
\hline 786 & $18^{1} ; 21^{2}$ & 405 \\
\hline 850 & $21^{10}$ & 504 \\
\hline 865 & $20^{1} ; 21^{6}$ & 3291 \\
\hline 871 & $18^{1} ; 21^{3}$ & 1045 \\
\hline 950 & $20^{1} ; 21^{7}$ & 904 \\
\hline 956 & $18^{1} ; 21^{4}$ & 1683 \\
\hline 961 & $16^{1} ; 21^{2}$ & 817 \\
\hline 965 & $20^{2} ; 21^{3}$ & 201 \\
\hline 966 & $15^{1} ; 21^{1}$ & 713 \\
\hline 1037 & $17^{1} ; 21^{3}$ & 398 \\
\hline & & \\
\hline
\end{tabular}

Figure 12. The theoretical onset of the $X^{2} A^{\prime}$ ionic state using anharmonic frequencies. Use of a half-width at half maximum of $10 \mathrm{~cm}^{-1}$ (in red) shows that several progressions occur, with the intensity passing from one series to a slightly higher wavenumber series. These are accumulated into single peaks when the linewidth is $20 \mathrm{~cm}^{-1}$ (in blue). 


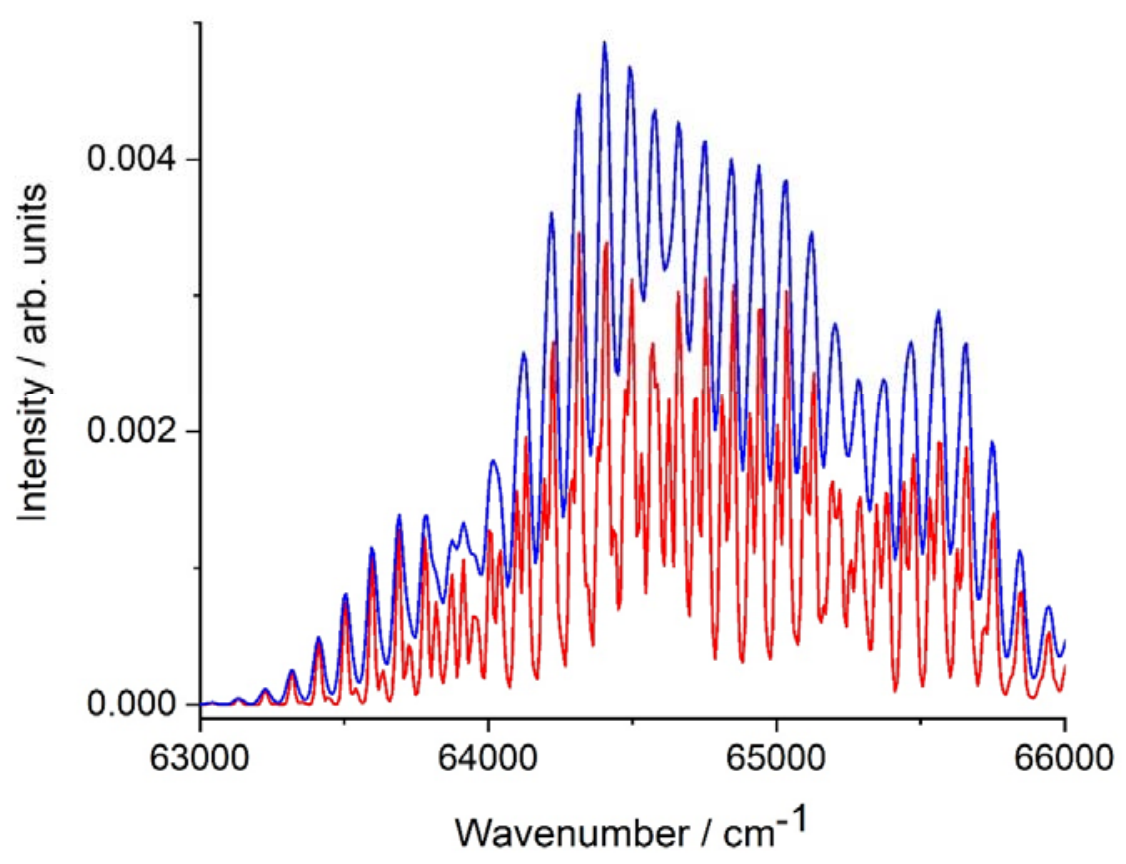

4. Analysis of the lowest UV absorption band of CHT. The absorption band lying between 4 and $5.5 \mathrm{eV}$ appears to be almost featureless, except for the set of sharp peaks on its trailing edge; these are attributable to the 3s-Rydberg state as discussed above. The width, exceeding $1.722 \mathrm{eV}$, implies considerable geometric change on excitation. The band was closely fit by an asymmetric Gaussian function, shown in the supplementary material as SM7; the regular residuals (RR) were processed separately. The fit function defines the onset peak maximum intensity at the vertical excitation energy (VEE) as 4.65 (1) eV. The RR of the low energy side (leading edge) show extremely weak fine structure, not previously observed, and shown in the supplementary material as SM8. We have extracted the data points for this region and found 17 peaks starting at $3.876 \mathrm{eV}\left(31265 \mathrm{~cm}^{-1}\right)$; the mean separation is $48 \mathrm{~cm}^{-1}$ with $\mathrm{SD} 6$ $\mathrm{cm}^{-1}$. The lowest calculated normal mode for $\mathrm{S}_{1}$ is $74.8 \mathrm{~cm}^{-1}$, is discussed below. This suggests that these two frequencies relate to different phenomena, since we would expect to calculate a low value rather than a high one, in the light of the Rydberg state above. 
Our spectral analysis was performed using the B3LYP functional within the TDDFT method. The lowest spectral band $\left(\mathrm{S}_{1}\right)$, assigned to a valence state ${ }^{1} \mathrm{~A}^{\prime \prime}\left(11 \mathrm{a}^{\prime} 8 \mathrm{a}^{\prime /}\right)$, in agreement with earlier work, ${ }^{16-19}$ was calculated at $4.527 \mathrm{eV}$ in $\mathrm{Cs}$ symmetry. Its relatively large oscillator strength, $\mathrm{f}(\mathrm{r}) 0.0986$ (Table II) indicates an allowed process.

The $\mathrm{S}_{1}$ theoretical AEE was effectively unchanged at $4.618 \mathrm{eV}$ when s-Rydberg basis functions were present in the similar calculation, and the leading configuration is the same; however, the presence of smaller amounts of $11 \mathrm{a}^{\prime} 12 \mathrm{a}^{\prime}$ and $10 \mathrm{a}^{\prime} 12 \mathrm{a}^{\prime}$ configurations, both of which are Rydberg excitations, suggests that there is some Rydberg character to the band. The partial spectral overlay of $\mathrm{S}_{1}$ with the $3 \mathrm{~s}-\mathrm{Rydberg}$ state makes this probable; hence neither state may be pure valence or Rydberg.

The active set of harmonic frequencies for the $\mathrm{S}_{1}$ state are shown in Table VI. Detailed analysis by Franck-Condon (FC), Herzberg-Teller (HT), and their combined methods (FCHT), given in Fig. 13 and Table VII, is limited to values close to the onset. The calculated Franck-Condon (FC) envelope shows a series of groups of vibrational states, where hot bands are dominant. Several mid-range modes up to $1500 \mathrm{~cm}^{-1}$ are apparent; the absence of mode 21, which is again very low $\left(75 \mathrm{~cm}^{-1}\right)$ and several of the highest frequencies including mode $1\left(3341 \mathrm{~cm}^{-1}\right)$ in these theoretical analyses is notable. There is a small but relatively constant HT component in $\mathrm{S}_{1}$, which is a largely a FC band. We have been unable to fit these vibrational analyses to the nearly smooth spectral band, even using a Gaussian peak with FWHM of $400 \mathrm{~cm}^{-1}$. However, the absence of both fluorescence and vibrational structure on this $\mathrm{S}_{1}$ band, even in a $12^{\circ} \mathrm{K}$ matrix, reported in reference 25 , shows the $S_{1}$ lifetime is very short, and estimated at $114 \times 10^{-15}$ seconds. ${ }^{21,22}$ This leads to a highly excited ground state by internal conversion ${ }^{21,22}$ in the overall process $\mathrm{S}_{0} \rightarrow \mathrm{S}_{1} \rightarrow \mathrm{S}_{0}^{*}$.

Figure 13. The CHT UV band, including the hot-band region inset, with combined Franck-Condon and Herzberg-Teller (FCHT) contributions as red bars. These bars are converted to Gaussian functions with Half-Widths at Half-Maximum of 70 (blue) and 400 (olive) $\mathrm{cm}^{-1}$. A very much wider Gaussian would be necessary to generate the 
observed smooth spectrum from the set of states in red. The insert at low energy is the theoretical hot band profile.

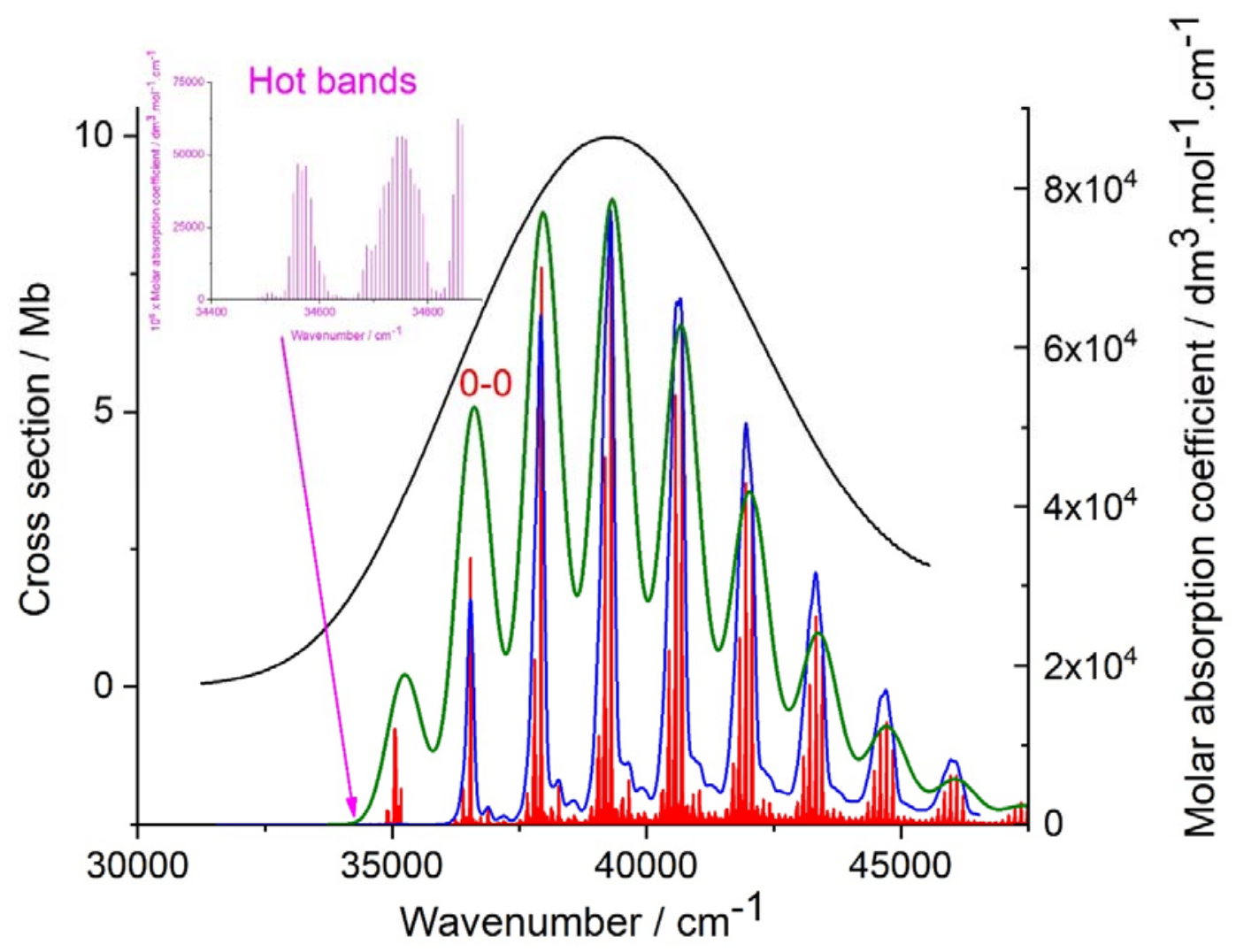

Table VI. The active $a^{\prime}$ harmonic frequencies $\left(\mathrm{cm}^{-1}\right)$ for the lowest singlet state $\left(\mathrm{S}_{1}\right)$ in $\mathrm{C}_{\mathrm{s}}$ symmetry.

\begin{tabular}{|c|c|c|}
\hline 173 & 936 & 1050 \\
\hline 447 & 972 & 1261 \\
\hline 569 & 995 & 1383 \\
\hline 706 & & \\
\hline
\end{tabular}

Table VII. Vibrational analysis of the lowest singlet state in terms of Franck-Condon(FC) and FC combined with Herzberg-Teller (FCHT) intensities, using low to high frequency mode sequence in G-16.

\begin{tabular}{|c|c|r|r|c|}
\hline $\begin{array}{c}\text { Frequency } \\
/ \mathrm{cm}^{-1}\end{array}$ & State & $\begin{array}{c}\text { FCHT } \\
\text { Intensity }\end{array}$ & $\begin{array}{c}\text { FC } \\
\text { Intensity }\end{array}$ & FCHT/ FC \\
\hline 0 & $0^{0}$ & 45730 & 44910 & 1.018 \\
\hline 346 & $2^{2}$ & 2452 & 2409 & 1.018 \\
\hline 692 & $2^{4}$ & 300 & 295 & 1.018 \\
\hline 706 & $8^{1}$ & 76 & -- & -- \\
\hline 879 & $8^{1}+2^{1}$ & 181 & 178 & 1.019 \\
\hline 995 & $16^{1}$ & 65 & -- & -- \\
\hline 1001 & $4^{1}+6^{1}$ & 94 & 92 & 1.018 \\
\hline 1050 & $17^{1}$ & 77 & -- & -- \\
\hline
\end{tabular}




\begin{tabular}{|r|c|r|r|r|}
\hline 1109 & $12^{1}+2^{1}$ & 72 & 71 & 1.018 \\
\hline 1138 & $6^{2}$ & 317 & 311 & 1.018 \\
\hline 1146 & $14^{1}+2^{1}$ & 454 & 446 & 1.018 \\
\hline 1168 & $16^{1}+2^{1}$ & 421 & 413 & 1.018 \\
\hline 1261 & $21^{1}$ & 32980 & 28310 & 1.165 \\
\hline 1275 & $8^{1}+6^{1}$ & 48 & 47 & 1.018 \\
\hline 1382 & $23^{1}$ & 104900 & 105700 & 0.992 \\
\hline 1412 & $8^{2}$ & 159 & 156 & 1.018 \\
\hline 1492 & $14^{1}+2^{3}$ & 70 & 68 & 1.018 \\
\hline 1514 & $16^{1}+2^{3}$ & 68 & 66 & 1.018 \\
\hline 1607 & $21^{1}+2^{2}$ & 1965 & 1687 & 1.165 \\
\hline
\end{tabular}

5. Comparison of the VUV absorption for CHT with that predicted for NCD. This is an attempt to confirm that the observed VUV spectrum emanating from a CHT sample is indeed that of CHT and not norcaradiene (NCD). VEE results for the two spectra, predicted at the B3LYP TDDFT level, and shown in Figure 14, were obtained by using the aug-cc-pVTZ basis set. Each compound is at its equilibrium structure. Both envelopes were plotted using Gaussian functions with width $3000 \mathrm{~cm}^{-1}$, as in Figure 3. The aug-cc-pVTZ basis set, used to generate both Figs. 3 and 14, is largely for valence state prediction rather than for Rydberg states. But since the latter are generally low in oscillator strength, the main profile will arise from valence states.

The two spectra exhibit significant differences. The absorption maxima predicted for NCD near 6.8 and $8 \mathrm{eV}$ correspond to minima for CHT. Conversely, the CHT maximum corresponds to a minimum for NCD. Lastly, the lowest NCD band is even lower than that for CHT, and both are lower in energy than the experimental spectrum. Overall, the predicted CHT spectrum offers a much better fit than the NCD spectrum to the observed VUV spectrum from CHT. This suggests that CHT is not isomerizing to NCD in our gas phase VUV spectrum by a significant amount. The two spectra are sufficiently different, that the presence of NCD in the vapor of CHT, could probably be recognized in the VUV spectrum.

Figure 14. A comparison of the predicted VUV spectra of CHT (in black) and NCD (in red) using the TDDFT method, together with the aug-cc-pVTZ basis set; this leads to a total of 543 basis functions. 


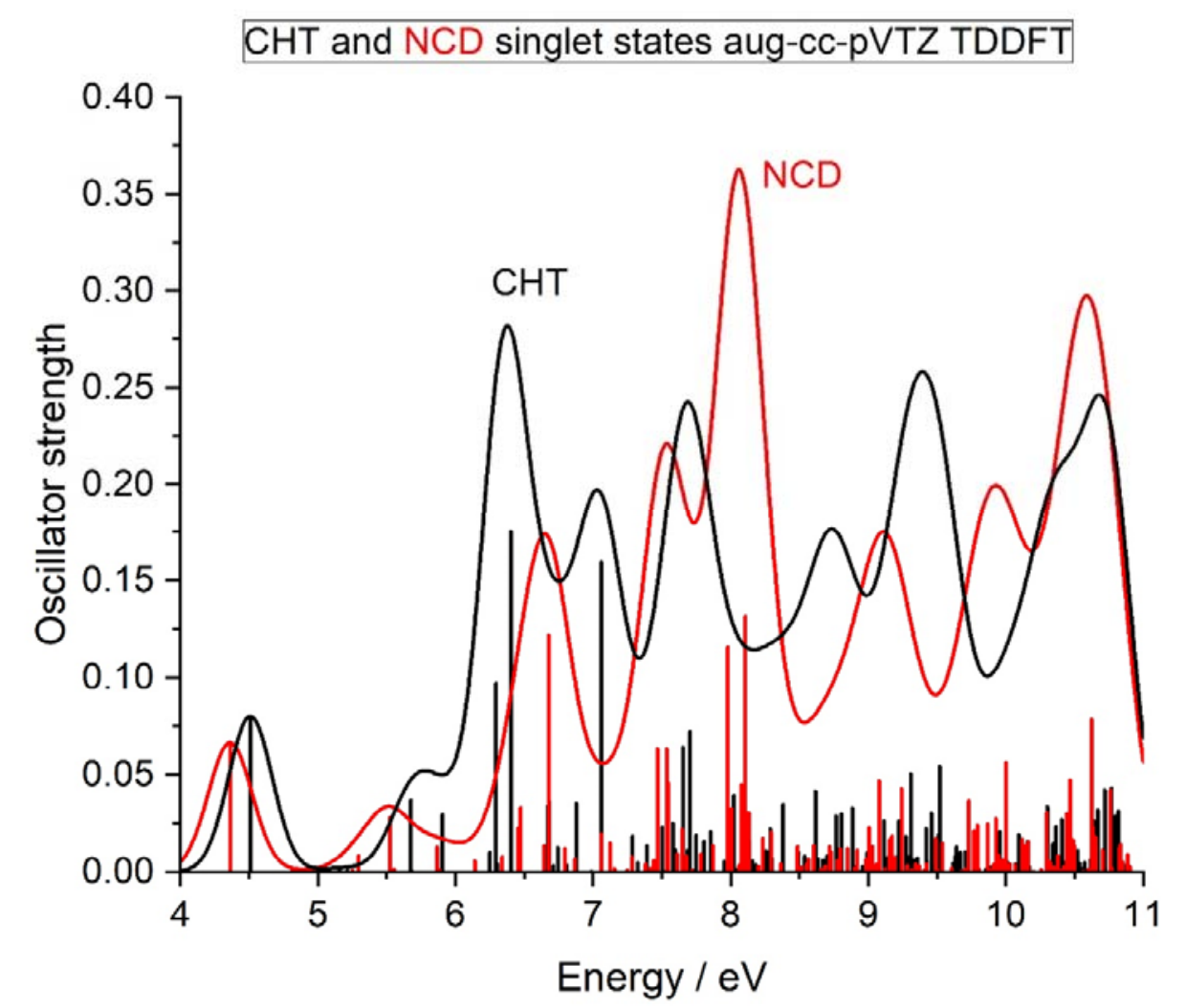

CONCLUSIONS.

Our theoretical manifold of excited singlet states for CHT at the TDDFT level gives a reasonable interpretation of the profile of the UV + VUV spectrum in the spectral range 4 to $10.8 \mathrm{eV}$. Most of the prominent peaks in the spectrum are a composite of several theoretical states. The $\mathrm{S}_{1}$ band onset, close to our lower limit of spectral range at $4.0 \mathrm{eV}$, is basically featureless except on both its leading and trailing edges. A considerable number of vibrational states were identified at both extrema, although those at the spectral onset are extremely weak; the vibration frequency doesn't match calculated values for $\mathrm{S}_{1}$. Our combined Franck-Condon and Herzberg-Teller profiles suggest that there should be an extensive set of vibrational states on $\mathrm{S}_{1}$ contrary to all studies to date. These analyses show the dominance of mid-range frequencies, but the negligible importance of the lowest frequency $\left(75 \mathrm{~cm}^{-1}\right)$ is notable. 
Previous studies, which have shown that $S_{1}$ has a particularly short life-time, suggest that this destroys the predicted vibrational structure.

Fine structure was also extracted from some higher energy VUV bands, by fitting a local energy range, subtraction of the intensity from the main absorption, and processing the regular residuals. The lowest state obtained by this method, already visible in the unprocessed spectrum, was clearly identified as the $3 \mathrm{~s}$-Rydberg state. It is a remarkable progression, with frequency $115 \mathrm{~cm}^{-1}$ and SD of only $5.5 \mathrm{~cm}^{-1}$ over at least 33 members; there is little anharmonicity. We believe that the apparently monotonic progression observed is accidental. The lack of apparent anharmonicity is surprising but is not unique, ${ }^{45}$ the propene (PP) and trans-but-2-ene (TBE) cations show strongly contrasting anharmonicity for their low frequency modes; PP is strongly anharmonic in contrast to TBE which is apparently remarkably harmonic. ${ }^{45}$ Since both these hydrocarbons are exhibiting $\pi$-ionizations, the overall situation is complex. Even the VUV multiplet of ethylene, has been reinterpreted to show a strongly harmonic progression together with an anharmonic torsional mode. ${ }^{48}$

The Rydberg state was analysed via the $\mathrm{X}^{2} \mathrm{~A}^{\prime}$ ionic state, thereby avoiding the complications with highly diffuse AOs. The only method which gave a positive vibration frequency for this state, was at the MP4(SDQ) level, although the value obtained $\left(88 \mathrm{~cm}^{-1}\right)$ is around $25 \%$ low when compared with the spectral value. Overall, the calculated profile for the $\mathrm{X}^{2} \mathrm{~A}^{\prime}$ ionic state gives a reasonable fit to the Rydberg state as expected, even though mismatches occur throughout the comparison. A variety of a' modes are involved, but the agreement was little changed by use of anharmonic frequencies.

This indicates that the MP4(SDQ) level of study for CHT is inadequate for this mode; a higher level treatment is needed for accurate reproduction of the experimental value, and this is beyond the scope of the present study. In support of these views concerning the necessary theoretical level of study, we have found that less sophisticated methods than the current ones, 
such as TDDFT, are even poorer and give negative frequencies for this mode in our hands. TDDFT is a single excitation level of CI.

The centroids of several of the higher energy Rydberg state bands, were extracted from the spectrum via their RR. Their energies were extrapolated to yield an experimental estimate of the vertical ionization energy which is relatively close to the experimental photoelectron value. In contrast to the ground state of CHT, which has a boat form with $\mathrm{C}_{\text {s }}$ symmetry, several singlet and triplet state structures are planar at the TDDFT single configuration level, although the structural differences relative to Cs symmetry are small. The planar forms are not confirmed by more rigorous methods, including a combined MP4(SDQ) and MCSCF study.

Theoretical studies using the TDDFT method at the vertical level, give a generally acceptable account of the UV+VUV spectral profile. Extremely diffuse Gaussian type orbitals enabled the theoretical adiabatic excitation energies of the $3 \mathrm{~s}, 4 \mathrm{~s}$ and $5 \mathrm{~s}$ Rydberg states to be determined; extrapolation of their energies gave a theoretical estimate of the adiabatic ionization energy; the experimental AIE has not been determined from the photoelectron spectrum.

Although there is direct evidence that $\mathrm{CHT}$ and $\mathrm{NCD}$ interconvert, the present study gave no evidence that NCD was present in the CHT sample. In order to differentiate the VUV spectra of the two compounds, we have generated predicted VUV spectra for both CHT and NCD under identical conditions, by using the TDDFT procedure. The predicted envelope for CHT in Figure 3, provides a reasonable reproduction of the experimental spectrum; the predicted spectrum for NCD is considerably different, and is likely to predict to a similar level of accuracy. In contrast to our work on the theoretical ionic ${ }^{2} \mathrm{~A}^{\prime \prime}$ states of CHT, where excited states of norcaradiene were obtained, none of the singlet or triplet states studied, undergo structural changes of that type.

\section{Acknowledgements}


We thank: (a) the ASTRID2 synchrotron facility, Aarhus University for a grant of beamtime. (b) Edinburgh Parallel Computing Centre for super-computing facilities (Cirrus), and the University of Edinburgh for similar access to Eddie3.

\section{Supplementary Material}

Additional information on the following is included:

1.The spectral method. 2.The lowest 100 singlet states with VEE and oscillator strengths, using the aug-ccpVTZ basis set and the B3LYP functional of DFT. 3.MP4(SDQ) equilibrium structures $\left(\mathrm{X}^{1} \mathrm{~A}^{\prime}=-270.7956841\right.$ a.u. $)$. 4. The $\mathrm{X}^{1} \mathrm{~A}^{\prime}$ and ${ }^{2} \mathrm{~A}^{\prime}$ state modes used in the FranckCondon analyses of the $\mathrm{X}^{2} \mathrm{~A}^{\prime}$ ionic state and applied to the 3s-Rydberg state. 5.The VUV spectrum accompanied by 150 singlet state TDDFT calculations, using the Rydberg enhanced version of the aug-cc-pVTZ basis set. The original set shown in Figure 9 are in red; the s- and pRydberg functions lead to the combined valence and Rydberg state calculations in blue and green respectively. In most states, the original red series have been overlaid by the s- and pRydberg state energies and oscillator strengths. For example, the single green band at $4.5 \mathrm{eV}$ has all three series of calculations overlaid with the p-set on top. In some cases, small shifts are observed as in the 5.5 to $6.0 \mathrm{eV}$ region. Very small differences are seen in the very intense peak at $7.1 \mathrm{eV}$, where all three colours are apparent. 6. Harmonic frequencies $\left(\mathrm{cm}^{-1}\right)$ for the lowest singlet state $\left(\mathrm{S}_{1}\right)$ in $\mathrm{C}_{\mathrm{S}}$ symmetry. 7.The planar forms of the HOMO and LUMO. 8. Asymmetric gaussian fit to the onset of the VUV spectrum. 9.Processing of regular residuals after fitting the onset to an asymmetric Gaussian function. 10. The full range of regular residuals after subtraction from the VUV spectral range.

\section{Declaration}

Data available on request from the authors. The data that support the findings of this study are available from the corresponding author upon reasonable request.

\section{REFERENCES}

1. M. H. Palmer, R. A. Aitken, M. Coreno, M. de Simone, C. Grazioli, S. Vrønning Hoffmann, and N. C. Jones, J. Chem. Phys. 152, 144301 (2020). https://doi.org/10.1063/1.5142268

2. M. H. Palmer, M. Coreno, M. de Simone, C. Grazioli, S. Vrønning Hoffmann, and N. C. Jones, J. Chem. Phys. 150, 194305 (2019). https://doi.org/10.1063/1.5096254

3. M. H. Palmer, T. Ridley, S.Vrønning Hoffmann, N. C. Jones, M. Coreno, M. de Simone, C. Grazioli, M. Biczysko, A. Baiardi, and P. Limão-Vieira, J. Chem. Phys. 142, 134302 (2015). doi: 10.1063/1.4916121

4. F. A. L. Anet, J. Am. Chem. Soc. 86, 458-460 (1964). https://doi.org/10.1021/ja01057a034

5. F. R. Jensen and L. A. Smith, J. Am. Chem. Soc. 86, 956-957 (1964). https://doi.org/10.1021/ja01059a065 
6. J. B. Lambert, L. J. Durham, P. Lepoutere, and J. D. Roberts, J. Am. Chem. Soc. 87, 38963899 (1965). https://doi.org/10.1021/ja01095a018

7. H. Günther and R. Wenzl, Z. Naturforsch., Teil B, 22, 389-399 (1967). https://doi.org/10.1515/znb-1967-0405

8. R. Wehner and H. Günther, Chem. Ber. 107, 3152-3153 (1974). https://doi.org/10.1002/cber.19741070938

9. D. I. Freedberg, M. Kopelevich, and F. A. L. Anet, J. Phys. Org. Chem. 14, 625-635 (2001). https://doi.org/10.1002/poc.410

10. M. B. Rubin, J. Am. Chem. Soc. 103, 7791-7792 (1981).

https://doi.org/10.1021/ja00416a018

11. R. Huisgen, Angew. Chem. Int. Ed. Engl. 9, 751-762 (1970).

https://doi.org/10.1002/anie.197007511

12. G. Maier, Angew. Chem. Int. Ed. Engl. 6, 402-413 (1967).

https://doi.org/10.1002/anie.196704021

13. A. A. Jarzęcki, J. Gajewski, and E. R. Davidson, J. Am. Chem. Soc. 121, 6928-6935 (1999). https://doi.org/10.1021/ja9844711

14. K. N. Klump and J. P. Chesick, J. Am. Chem. Soc. 85, 130-132 (1963). https://doi.org/10.1021/ja00885a003

15. D. C. Astholz, J. Troe, and W. Wieters, J. Chem. Phys. 70, 5107-5116 (1979). https://doi.org/10.1063/1.437352

16. P. M. Borrell, H. G Löhmannsröben, and K. Luther, Berichte der Bunsen-Gesellschaft 89, 274-275 (1985). https://doi.org/10.1002/bbpc.19850890317

17. P. M. Borell, H. G. Löhmannsröben, and K. Luther, Chem. Phys. Lett. 136, 371-376 (1987). https://doi.org/10.1016/0009-2614(87)80269-5

18. H. G. Löhmannsröben, and K. Luther, Zeit. fur Physikal. Chemie Neue Folge, 149,129-151 (1986)

19. P. J. Reid, M. K. Lawless, S. D. Wickham, R. A. Mathies, J. Phys. Chem., 98, 5597 (1994).

20. H-M. Steuhl, C. Bornemann, and M. Klessinger, Chem. Eur. J., 5, No. 8, 2404 (1999).

21. W. G. Dauben and R. L. Cargill, Tetrahedron, 12, 186 (1961).

22. R. Srinivasan, J. Am. Chem. Soc., 84, 3432 (1962).

23. F. W. E. Knoop, J. Kistemaker, and L. J. Oosterhoff, Chem. Phys. Lett. 3, $73-77$ (1969). https://doi.org/10.1016/0009-2614(69)80051-5 
24. R. P. Frueholz, R. Rianda, and A. Kuppermann, Chem. Phys. Lett. 55, 88-91, (1978). https://doi.org/10.1016/0009-2614(78)85140-9

25. N. L. Asfandiarov, S. A. Pshenichnyuk, A. S. Vorob'ev, E. P. Nafikova, V. K. Mavrodiev, I. I. Furlei, V. A. Dokichev, D. N.Platonov, and A. Yu. Belyy, Russian Journal of Physical Chemistry A 91, 915-920 (2017). https://doi.org/10.1134/S0036024417050041

26. Y. Valadbeigi and J.-F. Gal, Mol. Phys. 118, e1662507/1-e1662507/10 (2020), https://doi.org/10.1080/00268976.2019.1662507

27. Gaussian 16, Revision A.03, M. J. Frisch, G. W. Trucks, H. B. Schlegel, G. E. Scuseria, M. A. Robb, J. R. Cheeseman, G. Scalmani, V. Barone, G. A. Petersson, H. Nakatsuji, X. Li, M. Caricato, A. V. Marenich,J. Bloino, B. G. Janesko, R. Gomperts, B. Mennucci, H. P. Hratchian, J. V. Ortiz, A. F. Izmaylov, J. L. Sonnenberg, D. Williams-Young, F. Ding, F. Lipparini, F. Egidi, J. Goings, B. Peng, A. Petrone, T. Henderson, D. Ranasinghe, V. G. Zakrzewski, J. Gao, N. Rega,G. Zheng, W. Liang, M. Hada, M. Ehara, K. Toyota, R. Fukuda, J. Hasegawa, M. Ishida, T. Nakajima, Y. Honda, O. Kitao, H. Nakai, T. Vreven, K. Throssell, J. A. Montgomery, Jr., J. E. Peralta, F. Ogliaro, M. J. Bearpark, J. J. Heyd, E. N. Brothers, K. N. Kudin, V. N. Staroverov, T. A. Keith, R. Kobayashi, J. Normand, K. Raghavachari, A. P. Rendell, J. C. Burant, S. S. Iyengar, J. Tomasi, M. Cossi, J. M. Millam, M. Klene, C. Adamo, R. Cammi, J. W. Ochterski, R. L. Martin, K. Morokuma, O. Farkas, J. B. Foresman, and D. J. Fox, Gaussian, Inc., Wallingford CT, 2016.

28. (a) M. J. Frisch, M. Head-Gordon, and J. A. Pople, Chem. Phys. Lett. 166, 275 (1990). https://doi.org/10.1016/0009-2614(90)80029-D (b) M. J. Frisch, M. Head-Gordon, and J. A. Pople, Chem. Phys. Lett. 166, 281 (1990). https://doi.org/10.1016/0009-2614(90)80030-H

29. R. Krishnan and J. A. Pople, Int. J. Quantum Chem. 14, 91-100 (1978). https://doi.org/10.1002/qua.560140109

30. T. Yanai, D. Tew, and N. Handy, "A new hybrid exchange-correlation functional using the Coulomb-attenuating method (CAM-B3LYP)," Chem. Phys. Lett., 393 51(2004). DOI: $\underline{10.1016 / j . c p l e t t .2004 .06 .011}$

31. H.-J. Werner, P. J. Knowles, F. R. Manby, M. Schütz, P. Celani, T. Korona, R. Lindh, A. Mitrushenkov, G. Rauhut, K. R. Shamasundar, T. B. Adler, R. D. Amos, A. Bernhardsson, A. Berning, D. L. Cooper, M. J. O. Deegan, A. J. Dobbyn, F. Eckert, E. Goll, C. Hampel, A. Hesselmann, G. Hetzer, T. Hrenar, G. Jansen, C. Köppl, Y. Liu, A. W. Lloyd, R. A. Mata, A. J. May, S. J. McNicholas, W. Meyer, M. E. Mura, A. Nicklaß, D. P. O'Neill, P. Palmieri, K. Pflüger, R. Pitzer, M. Reiher, T. Shiozaki, H. Stoll, A. J. Stone, R. Tarroni, T. Thorsteinsson, M. Wang, and A. Wolf, MOLPRO Version 2012.1 a package of ab initio programs, 2012, see http://www.molpro.net/.

32. H.-J. Werner and P. J. Knowles, J. Chem. Phys. 82, 5053-5063 (1985). https://doi.org/10.1063/1.448627

33. P. J. Knowles and H.-J. Werner, Chem. Phys. Lett. 115, 259-267 (1985). https://doi.org/10.1016/0009-2614(85)80025-7 
34. R. J. Buenker and S. Krebs, in Recent Advances in Computational Chemistry 4 (1999), (Recent Advances in Multireference Methods), 1-29.

35. M. F. Guest, I. J. Bush, H. J. J. Van Dam, P. Sherwood, J. M. H. Thomas, J. H. Van Lenthe, R. W. A. Havenith, and J. Kendrick, Mol. Phys. 103, 719-747 (2005). https://doi.org/10.1080/00268970512331340592

36. J. Bloino, M. Biczysko, O. Crescenzi, and V. Barone, J. Chem. Phys. 128, 244105 (2008). https://doi.org/10.1063/1.2943140

37. V. Barone, J. Bloino, M. Biczysko, and F. Santoro, J. Chem. Theory Comput. 5, 540-554 (2009). https://doi.org/10.1021/ct8004744

38. J. Bloino, M. Biczysko, F. Santoro, and V. Barone, J. Chem. Theory Comput. 6, 12561274 (2010). https://doi.org/10.1021/ct9006772

39. T. H. Dunning, J. Chem. Phys. 90, 1007-1023 (1989). https://doi.org/10.1063/1.456153

40. R. A. Kendall, T. H. Dunning, and R. J. Harrison, J. Chem. Phys. 96, 6796-6806 (1992). https://doi.org/10.1063/1.462569

41. M. J. Frisch, J. A. Pople, and J. S. Binkley, J. Chem. Phys. 80, 3265-3269 (1984).

https://doi.org/10.1063/1.447079

42. F. Weigend and R. Ahlrichs, Phys. Chem. Chem. Phys. 7, 3297-3305 (2005).

https://doi.org/10.1039/B508541A

43. F. Weigend, Phys. Chem. Chem. Phys. 8, 1057-1065 (2006).

https://doi.org/10.1039/B515623H

44. M. H. Palmer, T. Ridley, S. Vrønning Hoffmann, N. C. Jones, M. Coreno, M. de Simone, C. Grazioli, M. Biczysko, A. Baiardi, and P. Limão-Vieira, J. Chem. Phys. 142, 134302 (2015); https://doi.org/10.1063/1.4916121

45. A. B. Burrill and P. M. Johnson, J. Chem. Phys., 115, 133 (2001).

46. W. G. Mallard, J. H. Miller and K. C. Smyth, J. Chem. Phys., 79, 5900 (1983).

47. M-K. Bahng, X. Xing, S. J. Baek, X. Qian and C. Y. Ng, J. Phys. Chem. A, 110, 8488 (2006).

48. A. Hazra, H. H. Chang and M. Nooijen, J. Chem. Phys., 121, 2125 (2004). 
Palmer et al Fig. 1

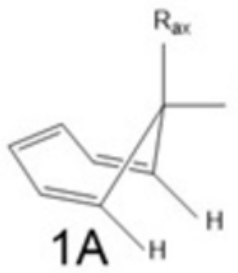

$\mathbf{R}_{\text {eq }}^{\prime}$
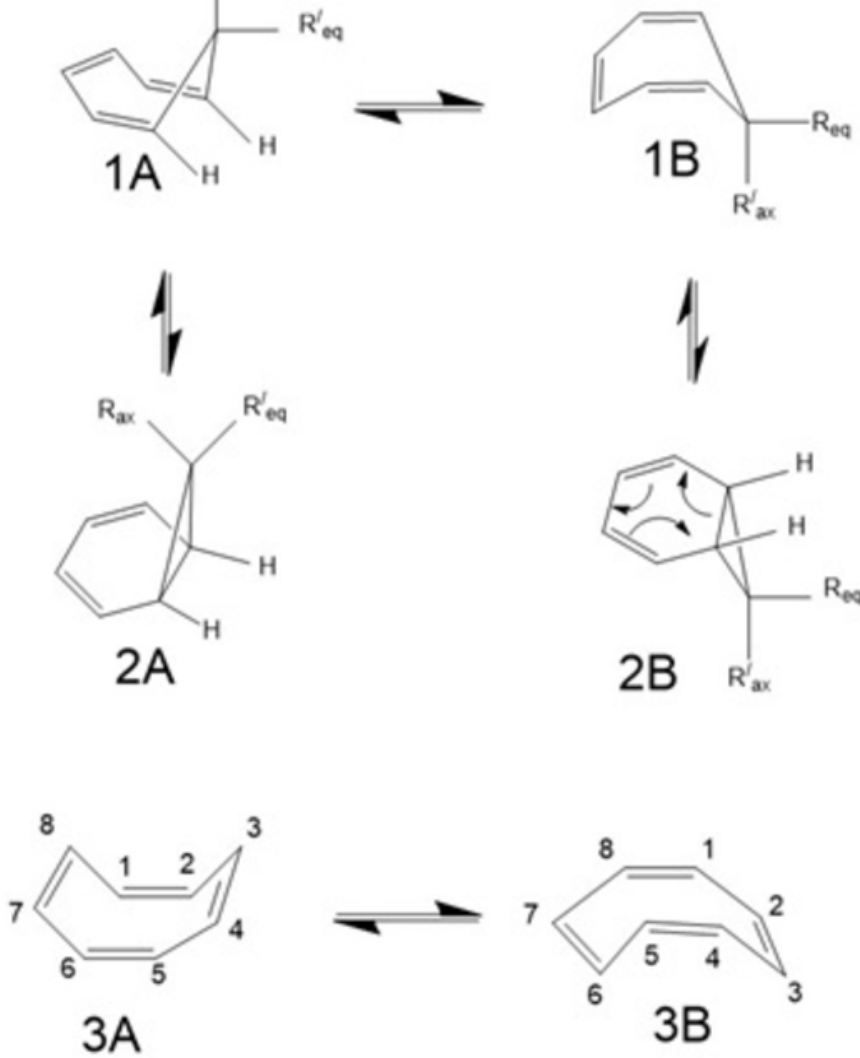


\section{Palmer et al Fig. 2}

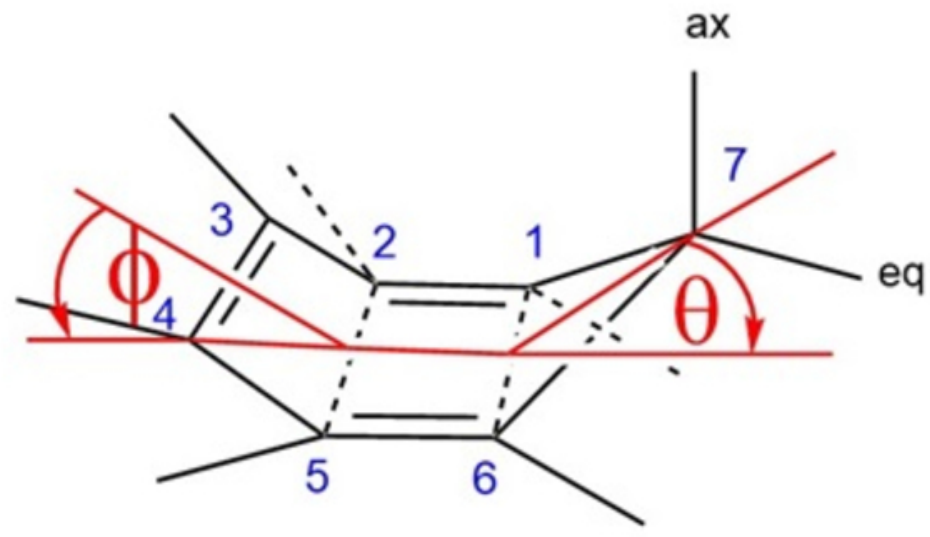




\section{Palmer et al Fig.3}

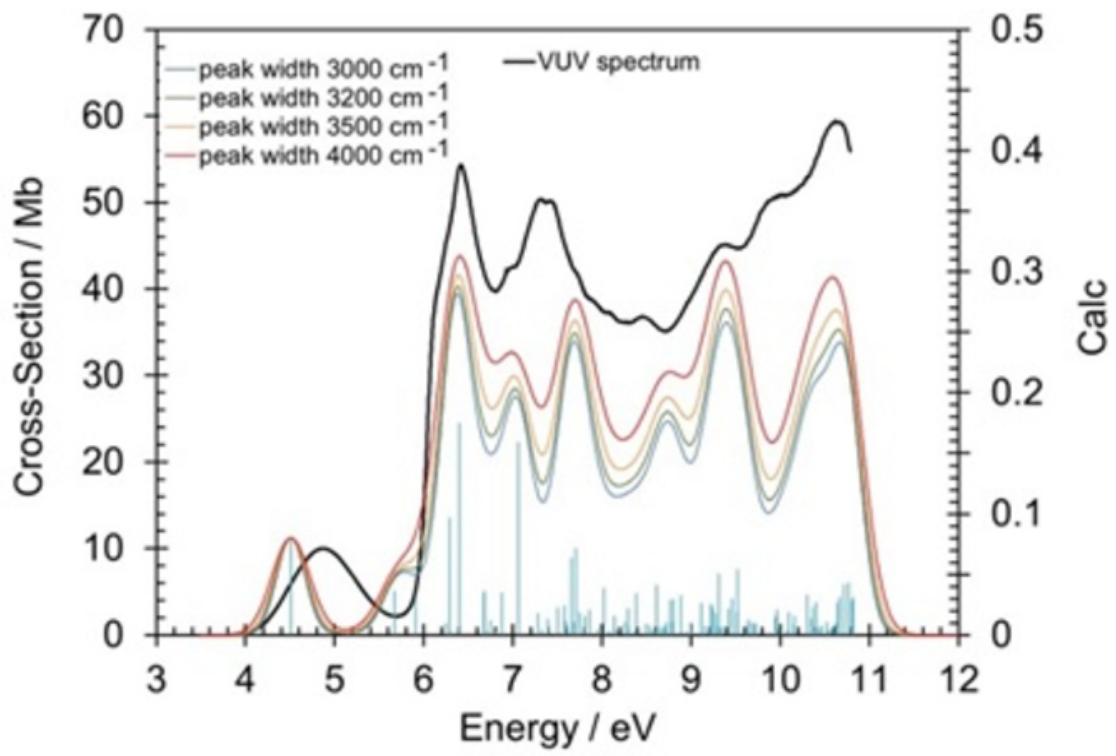




\section{Palmer et al Fig. 4}

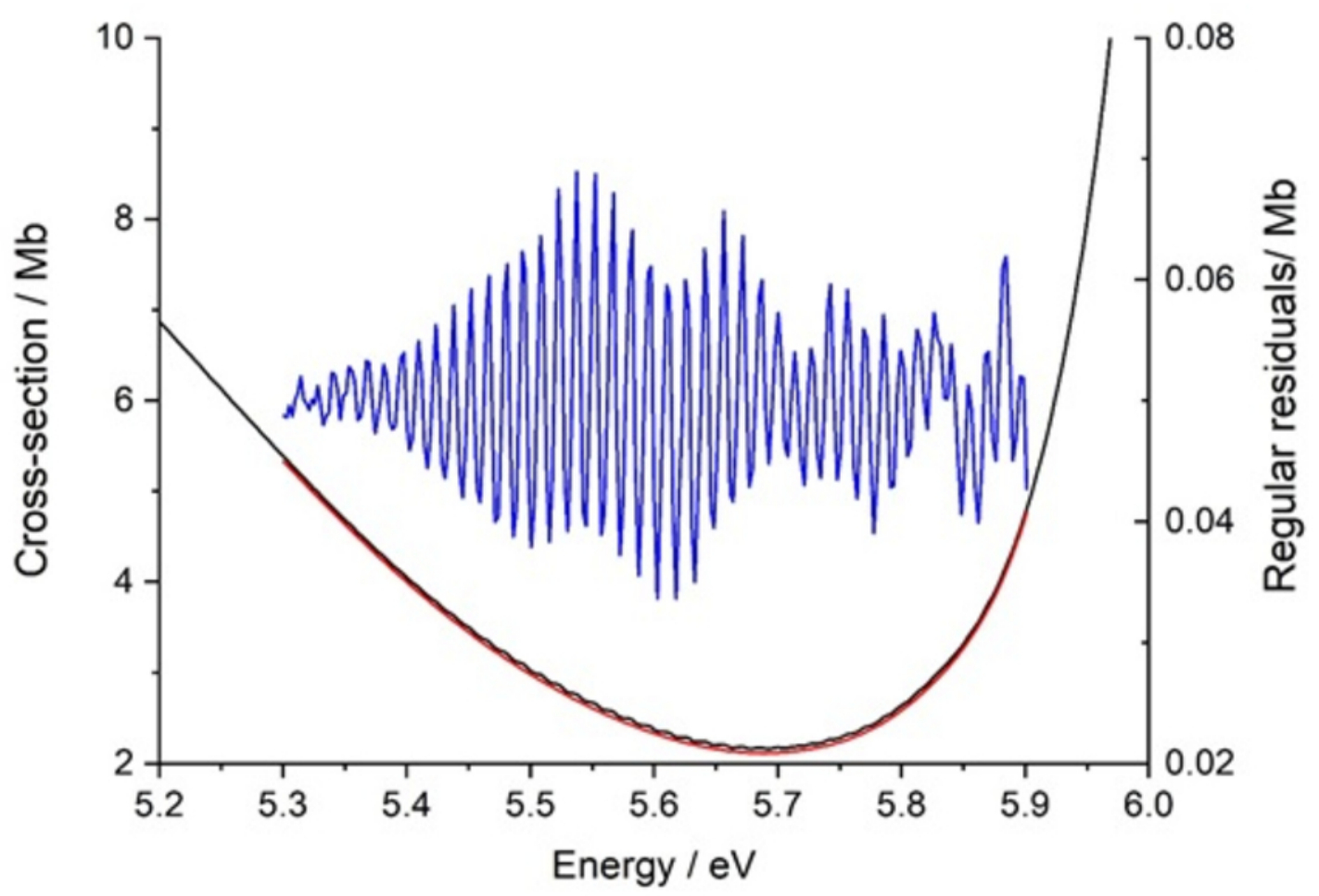




\section{Palmer et al Fig. 5}

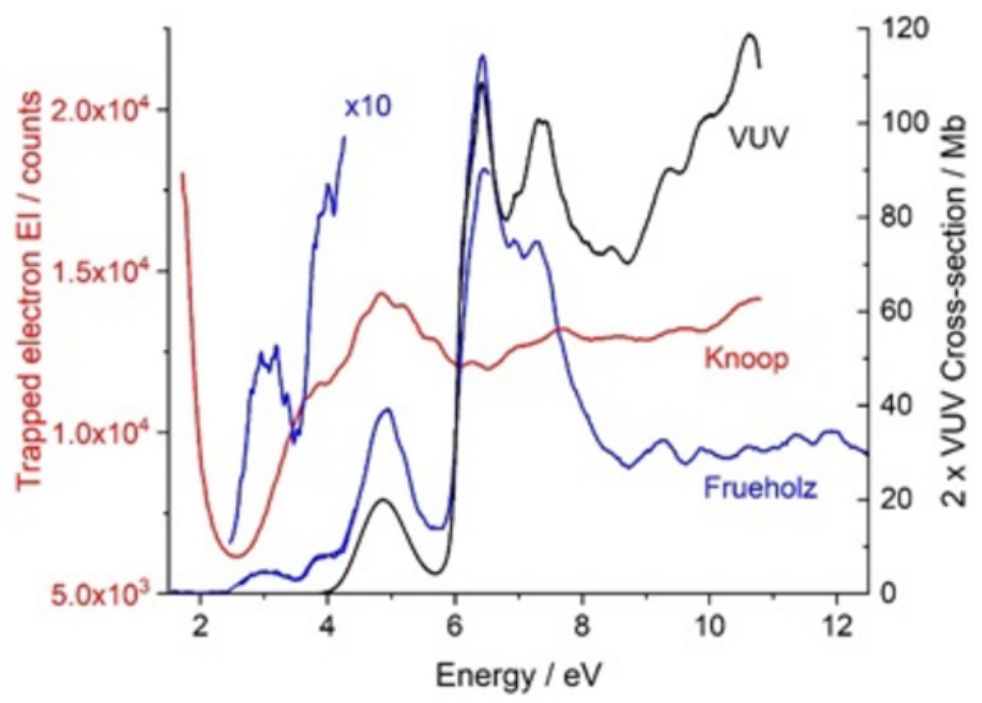


Palmer et al Figure 6

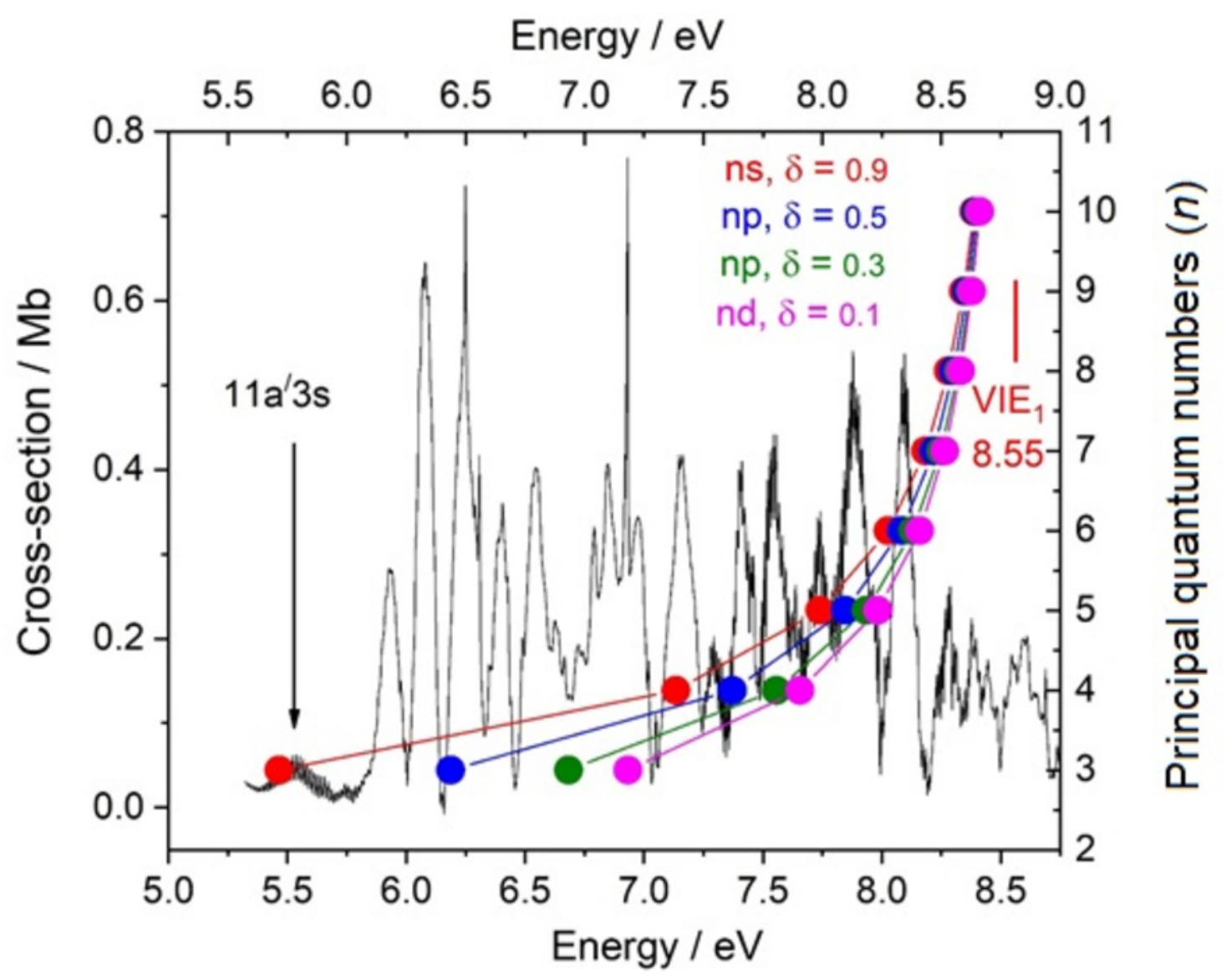




\section{Palmer et al Figure 7.}

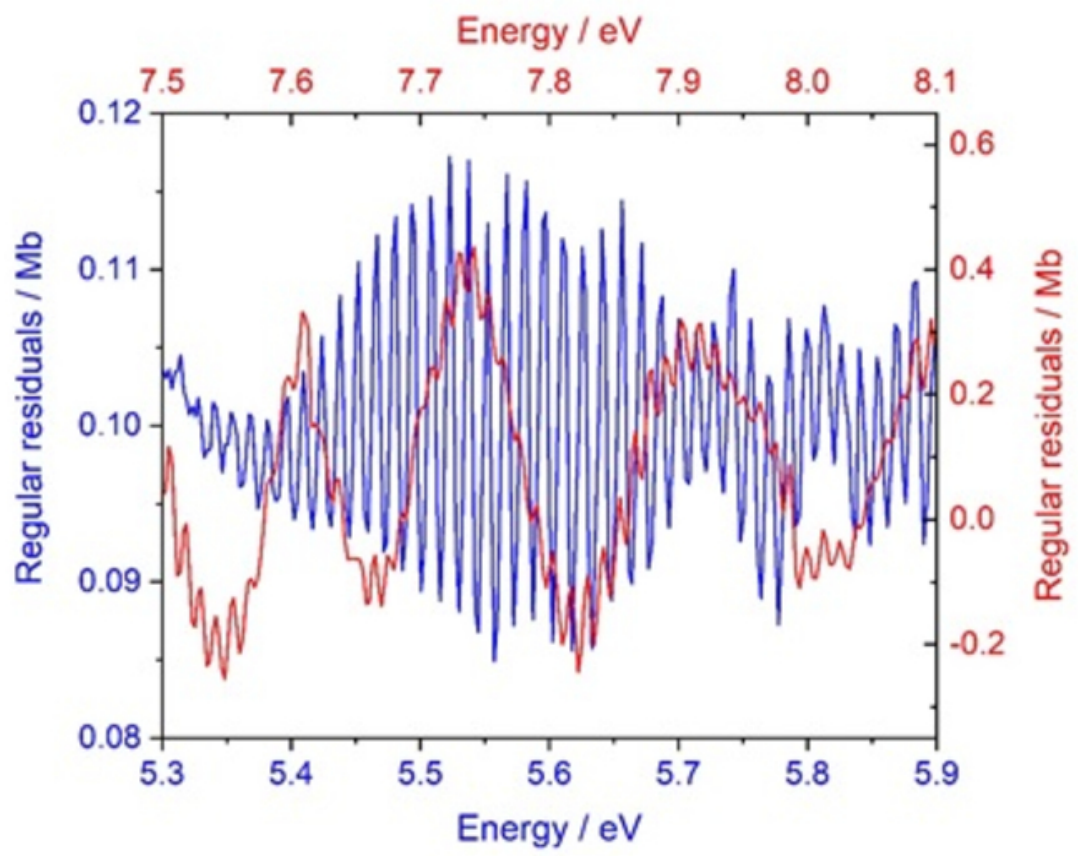




\section{Palmer et al Fig. 8}

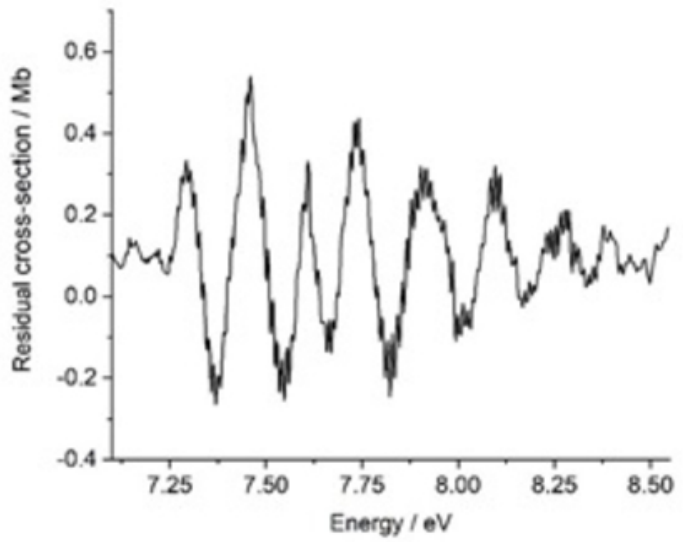




\section{Palmer et al Fig.9}

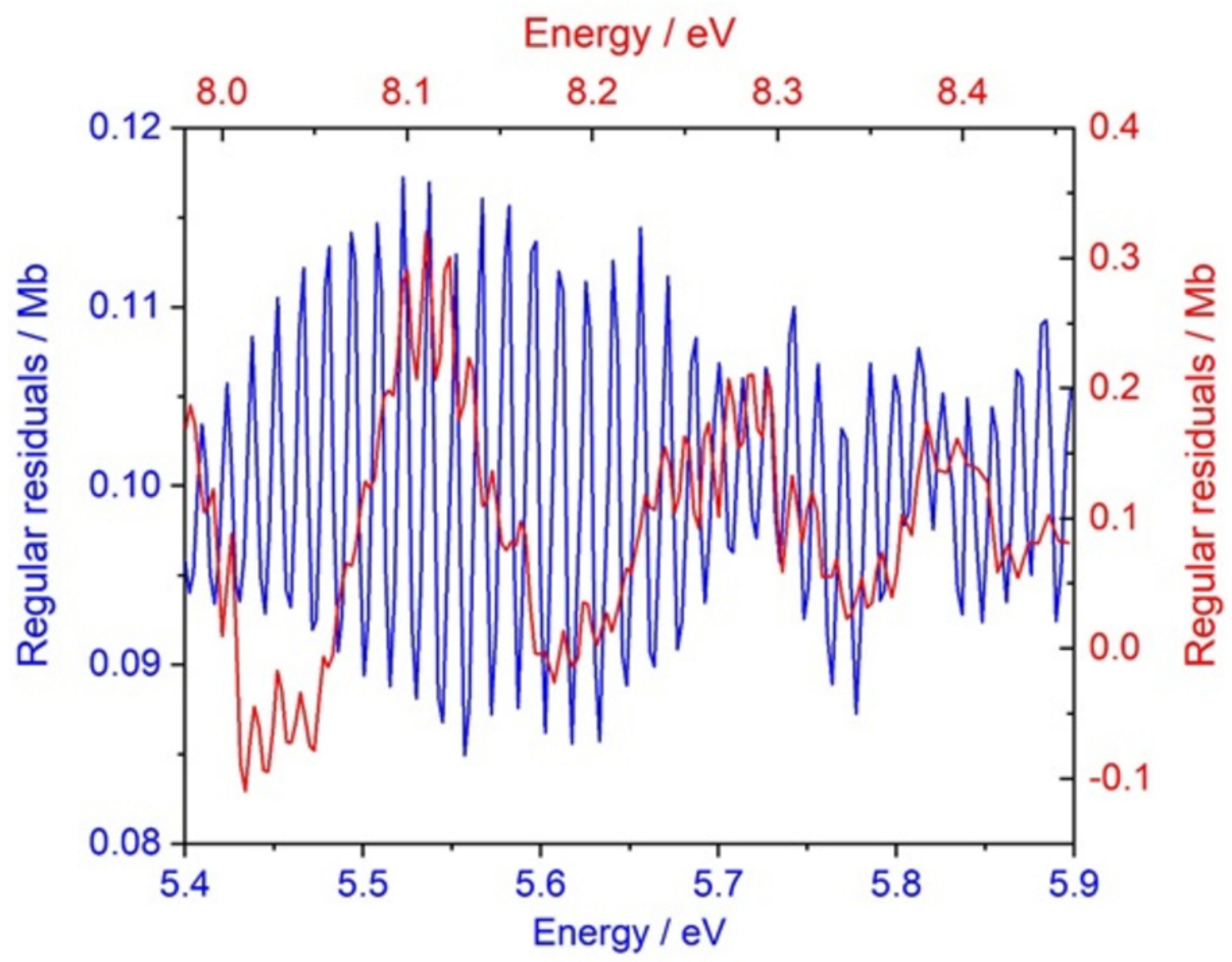




\section{Palmer et al Fig.10}

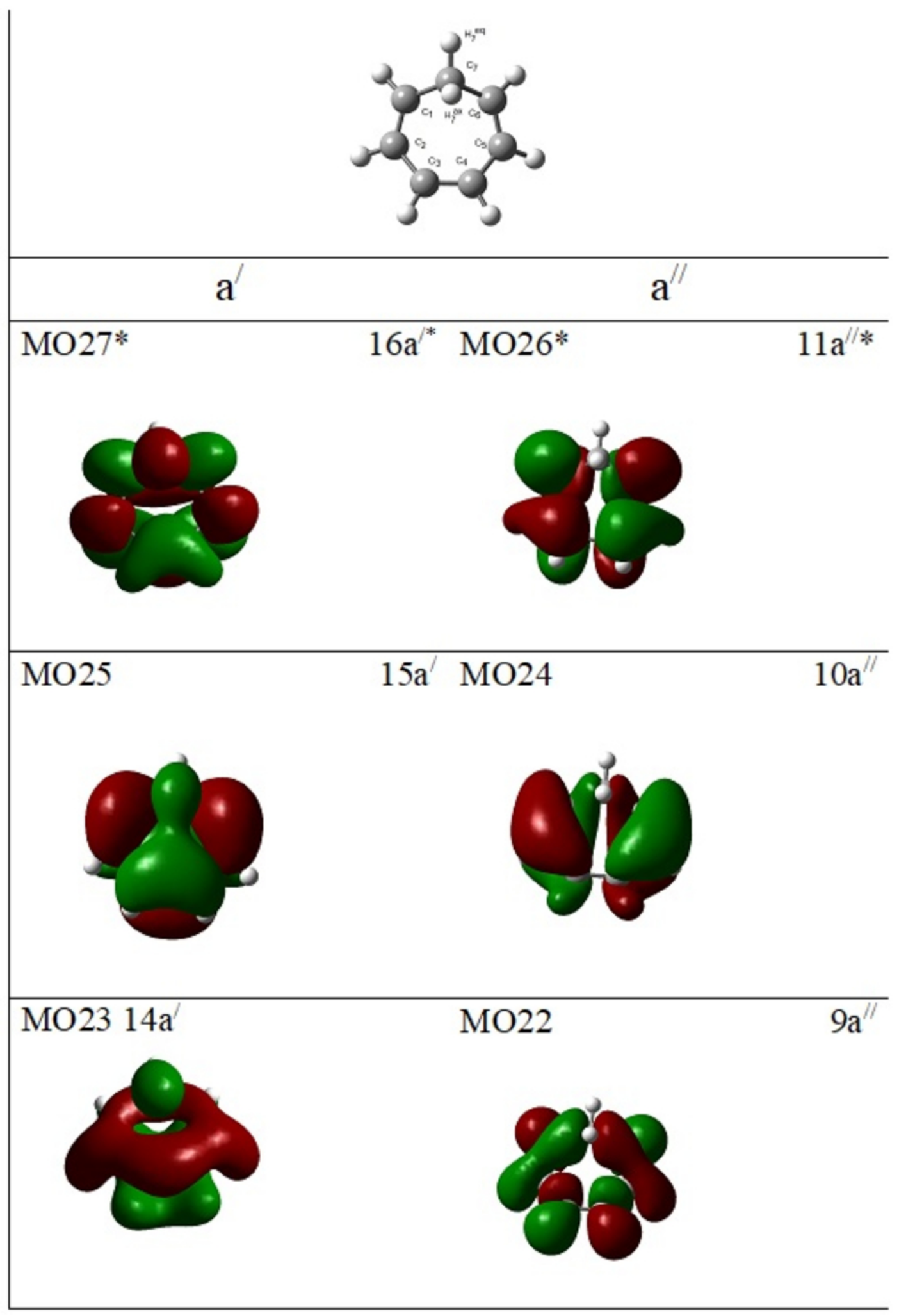


Palmer et al, Fig 11

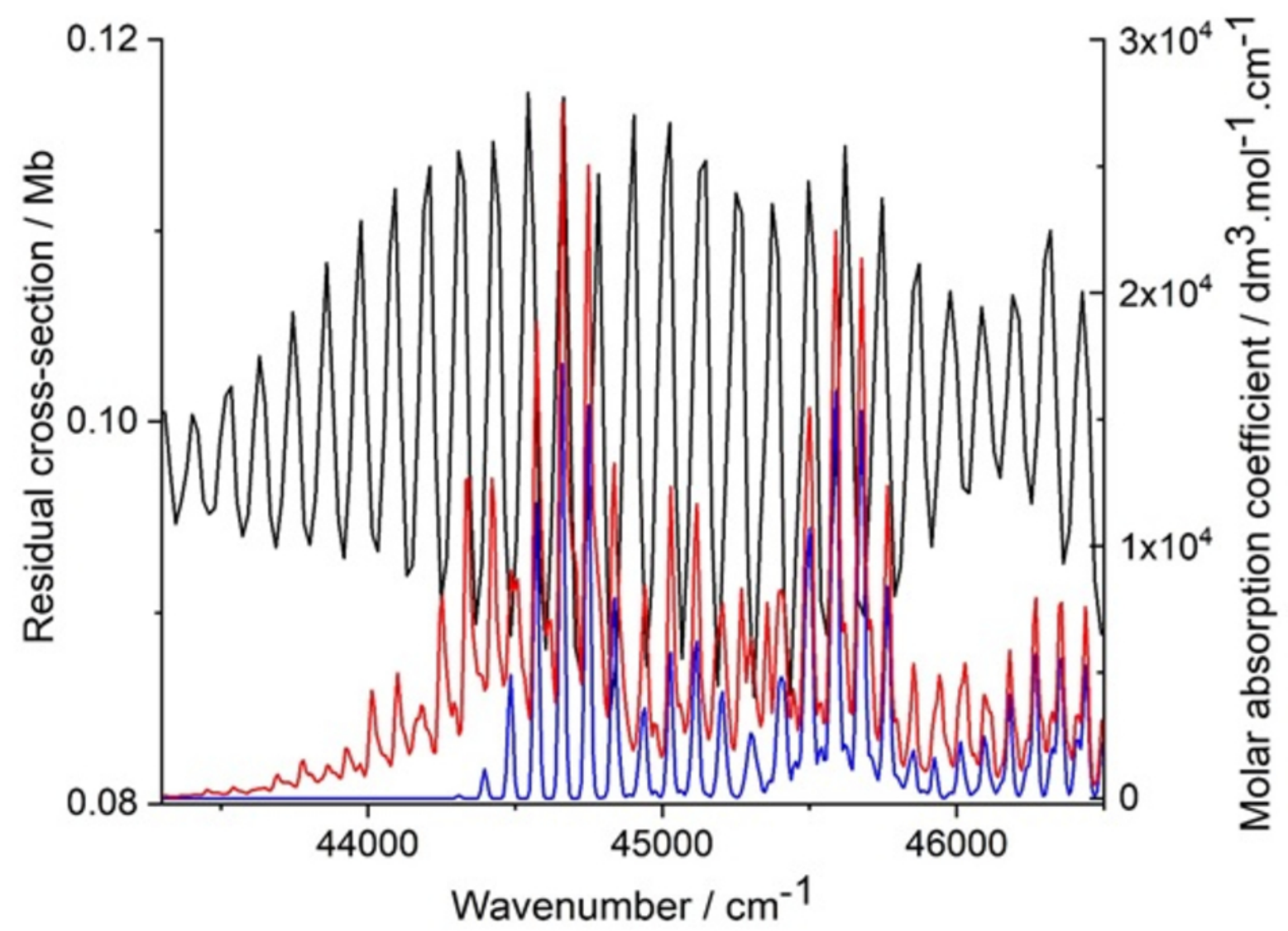


Figure 12. Palmer et al

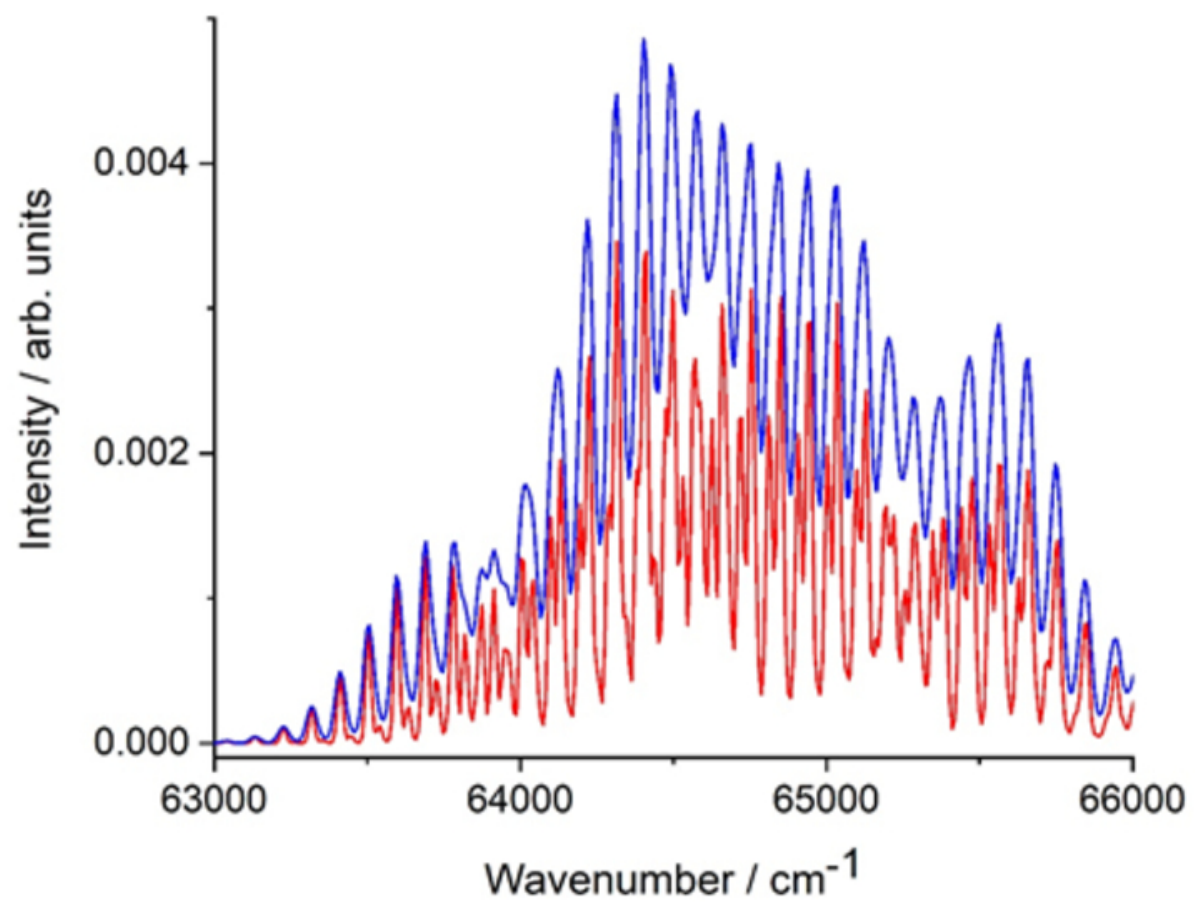




\section{Palmer et al, Fig13}

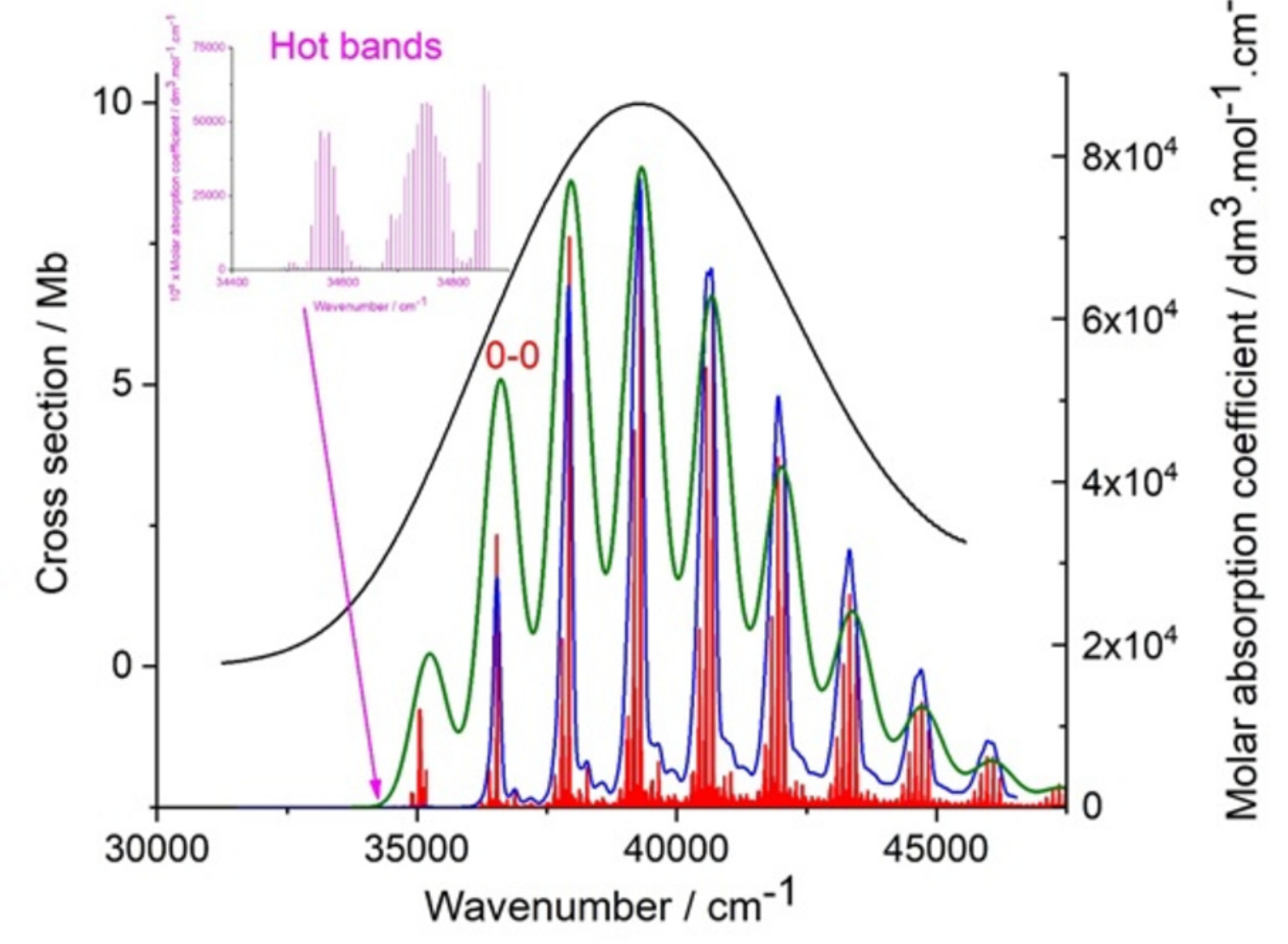




\section{Palmer et al, Fig 14}

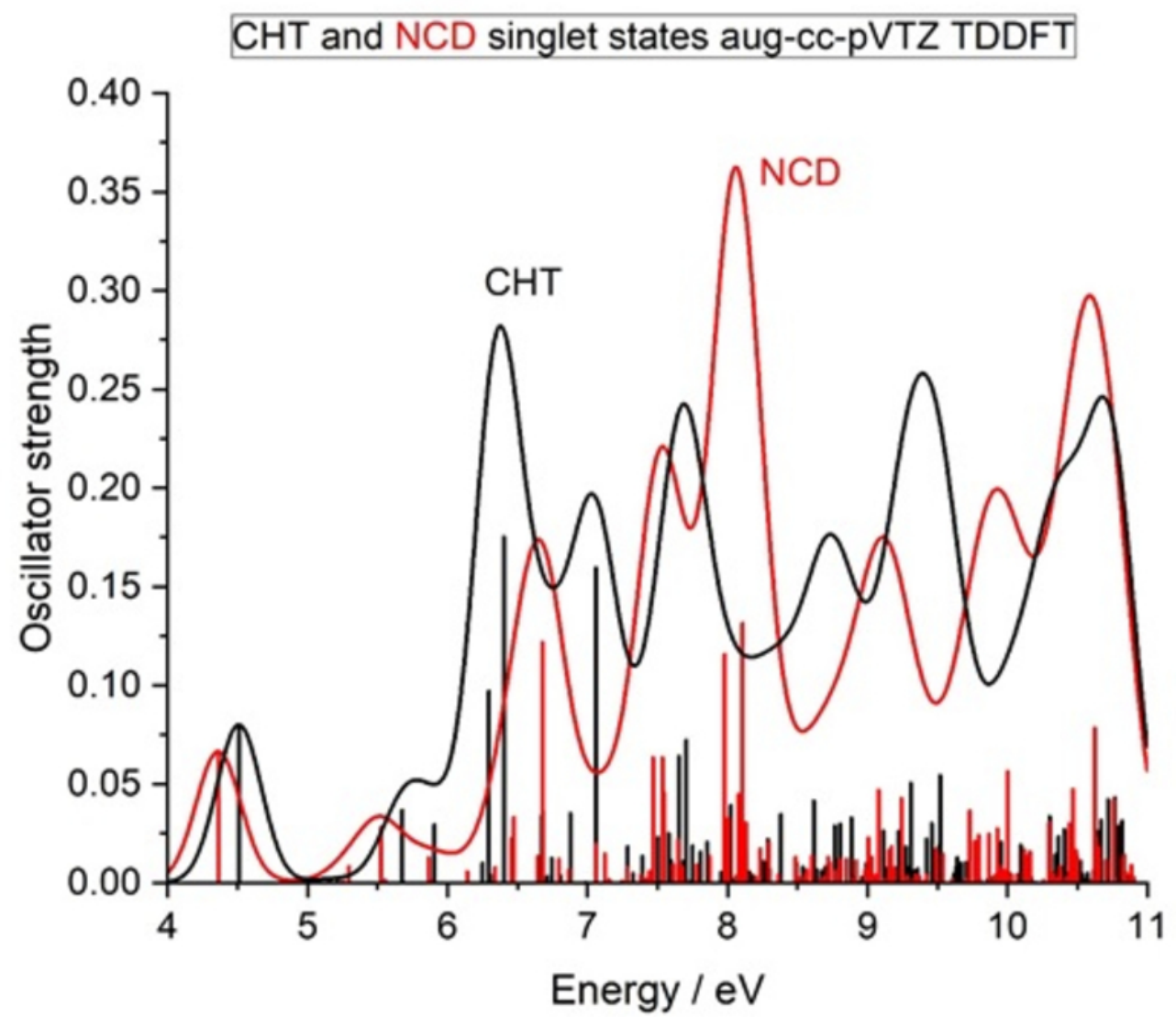

